
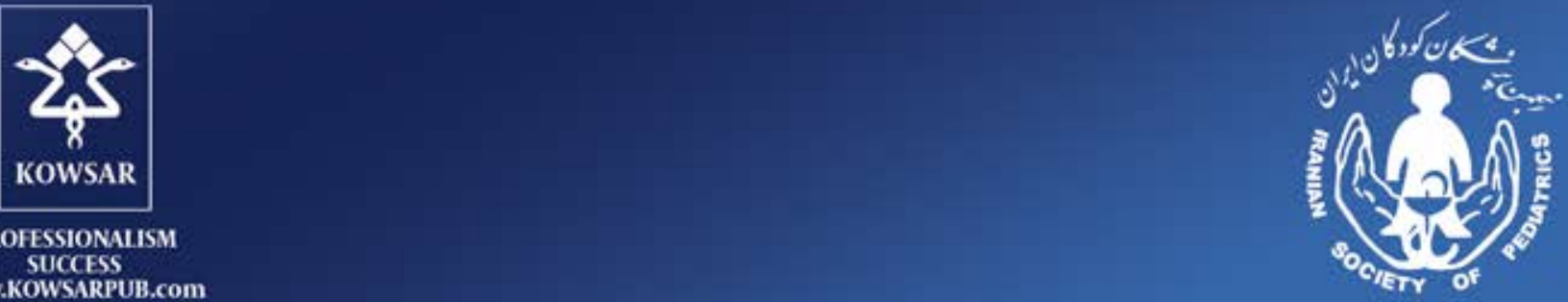

\title{
Journal of \\ COMPREHENSIVE PEDIATRICS
}

Official Quarterly Journal of Iranian Society of Pediatrics

ISSN: Print 2251-8150 Online 2251-8177

14-18 December 2020

Abstract of $16^{\text {th }}$ Congress of Pediatric Emergencies and Common Diseases 


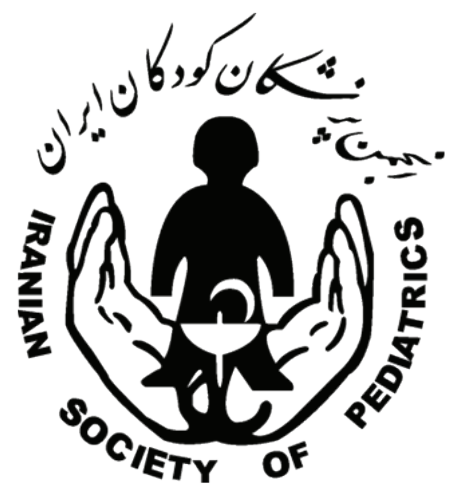

\section{Abstract of $16^{\text {th }}$ Congress of Pediatric Emergencies and Common Diseases}

14-18 December 2020

Tehran/Iran

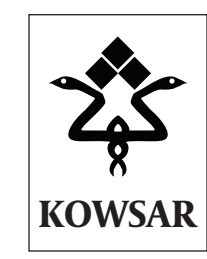

Kowsar Publishing Corp. 



\section{JOURNAL INFORMATION}

Journal of Comprehensive Pediatrics (J Compr Ped) is a quarterly official journal compiled by Iranian Society of Pediatrics (ISP).

\section{- AIMS AND SCOPE}

The open access journal of Comprehensive Pediatrics is an official journal of ISP, covering clinical and basic research, education, patients care, health economics, and policy to inform pediatricians. The journal welcomes all kinds of manuscripts and other scientific communications including original manuscripts, meta-analyses and reviews, health economic papers, debates, and consensus statements of clinical relevance of Pediatric fields.

\begin{abstract}
- CONTENT COVERAGE
Journal of Comprehensive Pediatrics is an authentic jounal and its content is devoted to selected compilation of the latest worldwide and interdisciplinary researches and reviews in the field of Pediatrics. In addition, consensus evidential reports not only highlight the new observations, original researches and results accompanied by innovative treatments and all other relevant topics, but also include highlighting disease mechanisms or important clinical observations and letters on articles published in journal.
\end{abstract}

\section{- SCIENTIFIC COLLABORATORS}

Journal of Comprehensive Pediatrics is published in co-operation with Iranian Society of Pediatrics, Tehran, Iran.

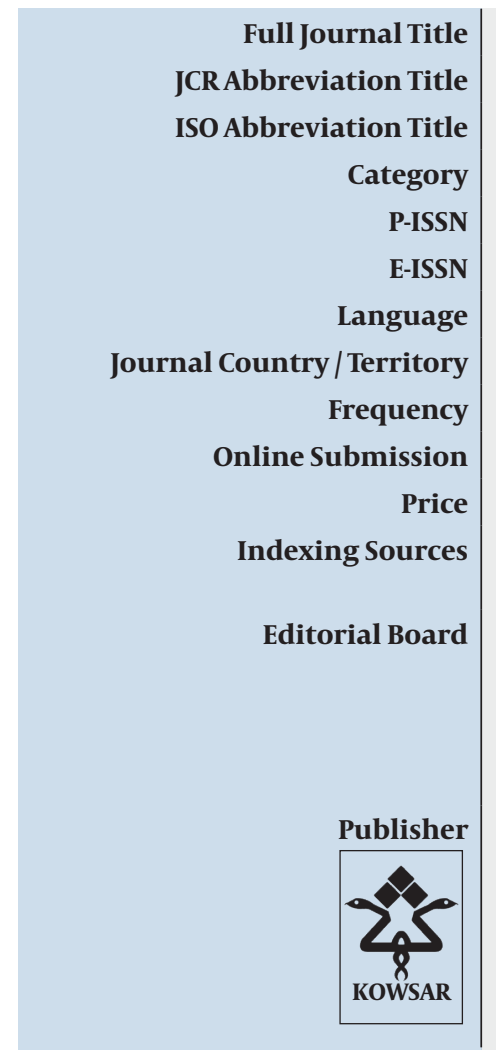

Journal of Comprehensive Pediatrics J COMPR PED

J. Compr. Ped.

Pediatric

2251-8150

2251-8177

English

Iran

Quarterly

sites.kowsarpub.com/jcp/

Online: Free

Scopus, Embase, Index Copernicus, Ulrichs Web, ROAD, Open J-Gate, DRJI, World Cat, Google Scholar, ISC, Ricest, Barakatkns, Magiran, SID

- Postal Address:

$3^{\text {rd }}$ Floor, No. 20, Bozorgmehr St., Valiasr Ave, Tehran, Iran; P. O. Box: 141693896; Tel: +98 $2166968317-8$ | Fax: +98 2166465828

- Electronic Address:

Website: sites.kowsarpub.com/jcp/ | E-mail: support@kowsarpub.com

Kowsarmedical

- Postal Address:

- Central Office: Kerkraderweg 13, 6416 CC, Heerlen, The Netherlands

Cell: +31 $615314198,+31455118301$ | Fax: +31 455118301.

- Electronic Address:

Website:www.kowsarpub.com | E-mail:info@kowsarpub.com 



\section{CONTENTS}

14-18 December 2020

- Introduction. $\ldots 1$

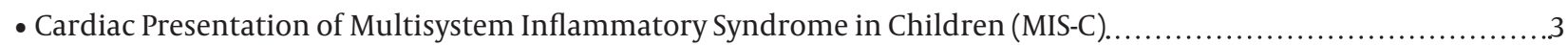

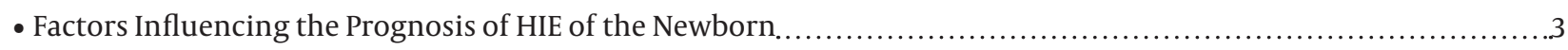

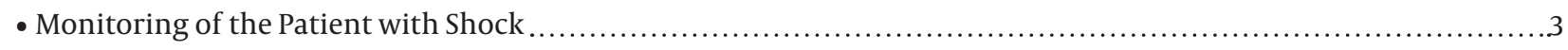

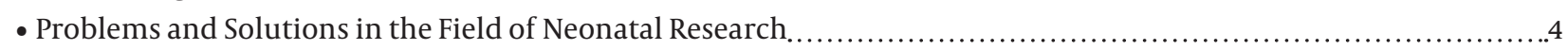

- Is Umbilical Cord Nucleated Red Blood Cell Count Related to a Premature Baby Prognosis?...........................4

- New Biomarkers in the Diagnosis of Hypoxic Ischemic Encephalopathy...........................................

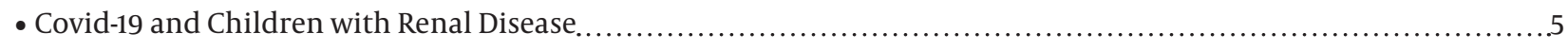

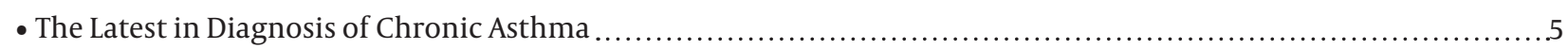

- Accuracy of Ultrasound in the Diagnosis of Skull Fractures in the Pediatric Emergency Department...................5

- Comparing Emergency Residents with Pediatric Residents for Their Skills of ECG Interpretation .....................5

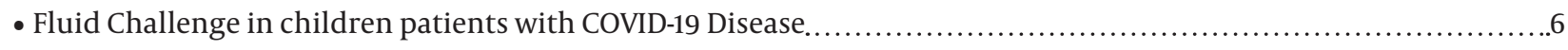

- Adolescent's Spiritual Health in COVID-19 Pandemic: A Qualitative Study........................................6

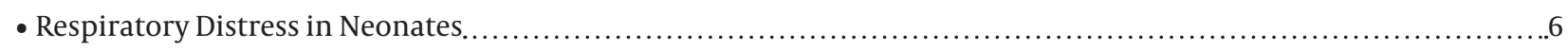

- Drug-Induced Pulmonary Toxicity: Lots of Drugs and Chemical Agents Can Cause Acute and Chronic Lung Injury.... .7

- Management of Shock in Multisystem Inflammatory Syndrome Associated with COVID-19 in Children (MIS-C)........7

- The Effect of Education on First Year Residents' Knowledge Regarding Hemovigilance System.........................7

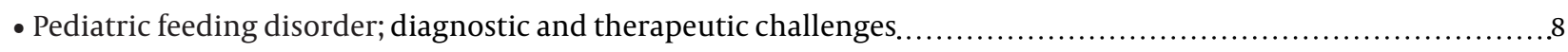

- Gastro esophageal Reflux Disease; diagnostic and therapeutic challenges .......................................... 8

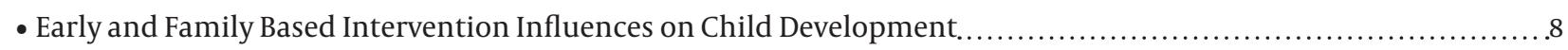

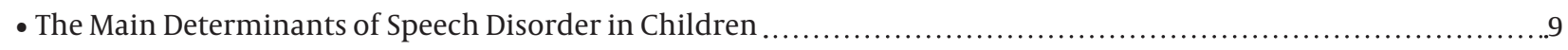

- Post COVID-19 Systemic Juvenile Idiopathic Arthritis in Children..................................................... 9

- Management of Children with Rheumatic Diseases in COVID-19 Outbreak ........................................9

- The Impact of Social Media \& Video Games on Neurological Development of Children...............................9

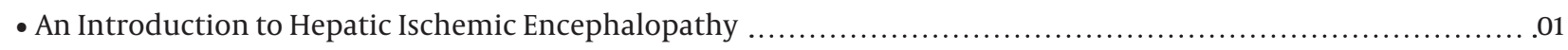

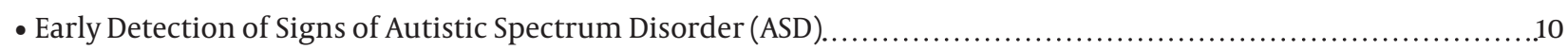

- Cow's Milk Protein Allergy Diagnostic and Therapeutic Challenges . .................................................

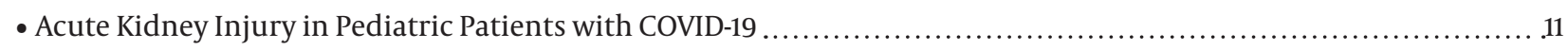

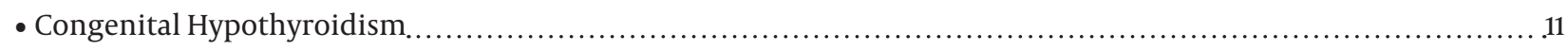

- Varicella Zoster Virus Immunoglobulin G in Immunosuppressed Children with Acute Lymphocytic Leukemia Under

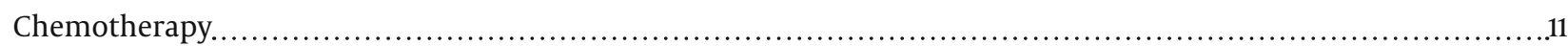

- Multi Organ Dysfunction in Infants with Hypoxic-Ischemic Encephalopathy........................................11

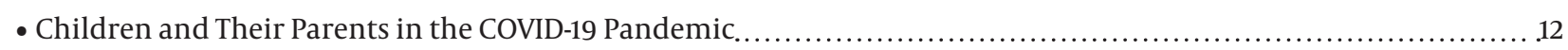

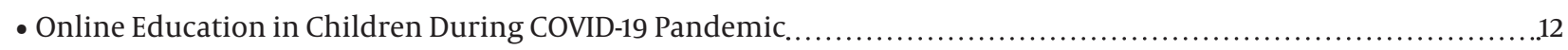

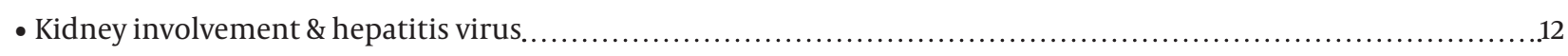

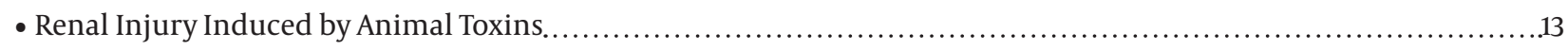

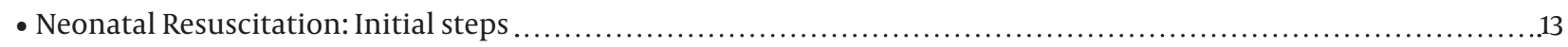

- Anti-Microbial Effect of Nigella Sativa Seed Extract Against Staphylococcal Skin Infection............................13 
- Treatment of Perinatal Asphyxia in Term and Late Preterm Infants . ...........................................13

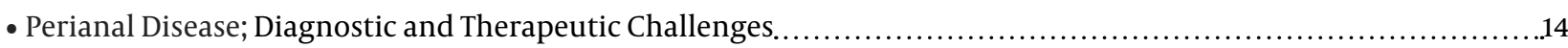

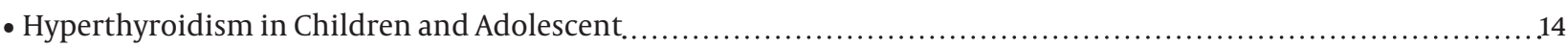

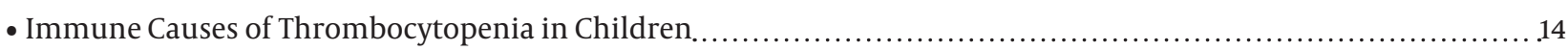

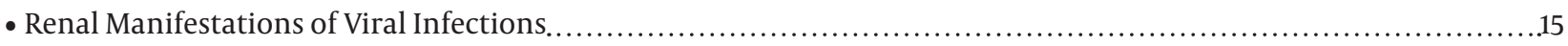

- Determination of Various Japanese Scores in Kawasaki Disease .................................................15

- Diagnostic Tests in Developmental Delays and Disorders in Children ..........................................15

- Serum Electrolytes and Trace Elements Disorders in Viral Diseases (Covid-19).....................................16

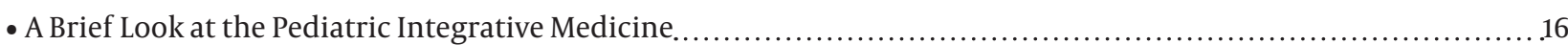

- Neonatal Mortality and Related Factors in Mahdiyeh Hospital through Seven Years ...................................16

- Chest Compression and Medications in Neonatal Resuscitation: 2020 American Heart Association Guidelines for Car-

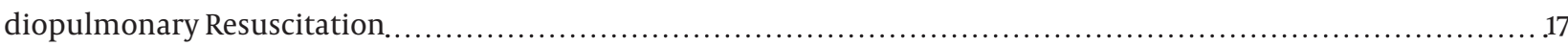

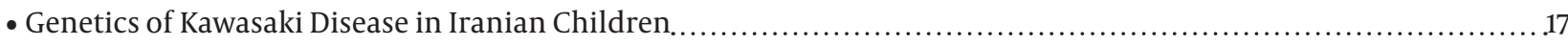

- Management of Spinal Muscular Atrophy New coordinated platform between patient and clinician in COVID-19 pan-

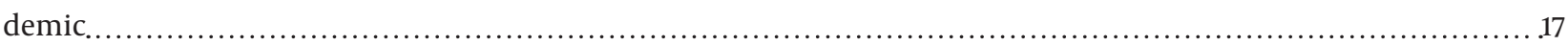

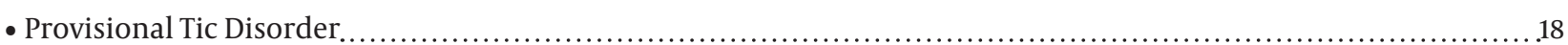

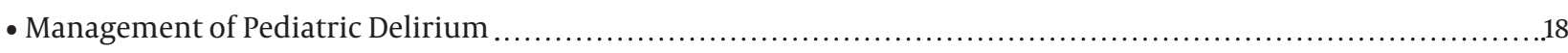

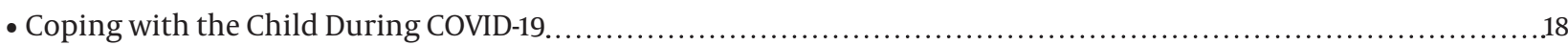

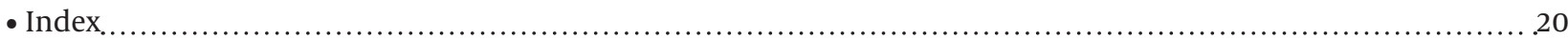




\section{INTRODUCTION}

\section{In the name of God}

Pediatric is one of greatest and ever-changing field of medicine. Common diseases and pediatric emergencies are the most important parts of this field. $16^{\text {th }}$ congress of pediatric emergencies and common diseases that is being held in Tehran/Iran 14-18 December cover the newest and hottest parts of pediatric with more than 15 years experience of this type of congress with leadership of pediatric department of Shahid Beheshti University of Medical Sciences. COVID 19 infection is one of new topic that was discussed in this congress comprehensively. The accepted abstracts of the congress are mentioned in supplementary number of Journal of Comprehensive Pediatrics.

Congress Chair

Mohammad Mehdi Nasehi MD; Associate Professor of Pediatric Neurology 



\section{-Cardiac Presentation of Multisystem Inflammatory Syndrome in Children (MIS-C)}

\author{
Fariba Alaei $^{1}$ \\ ${ }^{1}$ Pediatric Cardiology Department, Mofid Children's Hospital, Shahid Beheshti Univer- \\ sity of Medical Sciences, Tehran, Iran

\section{Abstract}

Coronavirus disease 2019 (COVID-19) is usually mild in children. Rarely, children are severely affected. Multisystem inflammatory syndrome in children (MIS-C) is an uncommon complication of COVID-19 often involving previously healthy older children and adolescents. It is thought to be the result of an abnormal immune response to the infection. Most affected children have negative polymerase chain reaction (PCR) and positive serology for SARS-CoV-2. Clinical presentation may include persistent fever, gastrointestinal symptoms and features like Kawasaki disease (KD) followed by shock or multisystem involvement. Inflammatory markers (erythrocyte sedimentation rate [ESR], C-reactive protein [CRP], D-dimer and procalcitonin) and cardiac biomarkers (Troponin, brain natriuretic peptide [BNP] or N-terminal proBNP [NT-proBNP]) are often elevated. Evidence of infection (PCR, antigen test or positive serology) or likely contact with COVID-19 patients should accompany. Myocardial injury, identified by the presence of cardiac troponin above the 99th percentile upper reference limit, is common. Possible causes include viral myocarditis, inflammation, hypoxia, stress cardiomyopathy and ischemia. Combinations of these mechanisms could be responsible for cardiac dysfunction. A minority of patients present cardiac symptoms or nonspecific symptoms. ST-segment and T-wave changes, arrhythmia or heart block may occur on electrocardiography (ECG) although most patients have non-specific ECG. Depressed left ventricular (LV) function, Coronary artery (CA) dilation or aneurysm, mitral valve regurgitation or pericardial effusion may occur on echocardiographic evaluation. Management depends on the clinical presentation and severity. All patients should receive empiric antibiotic and also antiviral therapy if evidence of active infection exists. Patients presenting shock are treated with intravenous fluid and vasoactive agents. Significant LV dysfunction necessitates supportive care to maintain hemodynamics, intravenous immune globulin (IVIG), diuretics and inotropes and rarely mechanical hemodynamic support. For patients with KD features, standard therapies are applied. Glucocorticoid therapy is recommended for severe or refractory shock, KD-like features with risk of IVIG resistance risk and persistent fever. Systemic anticoagulation is used for moderate to severe LV dysfunction and also older children and adolescents with moderate to severe MIS-C. The mortality rate is approximately 1 to 2 percent for MIS-C patients. Most children with cardiac involvement have recovery of ventricular function and resolution of arrhythmias at the time of hospital discharge, although mildly diastolic ventricular dysfunction usually persists in $20 \%$.

Keywords: Coronavirus Disease 2019, Multisystem Inflammatory Syndrome in Children, Myocardial Injury

\section{- Factors Influencing the Prognosis of HIE of the Newborn}

\author{
Mahta F .Bassir ${ }^{1}$
}

${ }^{1}$ Shohada General Hospital, Shahid Beheshti University of Medical Sciences, Tehran, Iran

\section{Abstract}

There are several degrees of HIE: mild, moderate to severe. In most cases, the injuries are sensorimotor. Although cognitive impairments without motor delay is not uncommon. Some factors are known as predictors of morbidity and severity of HIE: very low APGAR score at 10 or 15 minutes, prolongation and severity of neonatal neurologic syndrome, abnormal EEG, aEEG or evoked potential and also abnormal brain imaging. Hypoxic ischemic injury is one of the causes of low Apgar score and correlation with the severity of the injury is not always established. But a delay in the onset of spontaneous breathing of more than 20-30 minutes is usually associated with a higher risk of serious illness and high mortality rate. The new expanded Apgar score seems to be a more accurate instrument for evaluating the prognosis of HIE. The neonatal neurologic syndrome may be may manifested in the form of lethargy, apnea, feeding problems, hypertonia and seizures. Acidosis $(\mathrm{pH}<7)$ and abnormal fetal monitoring can also be valuable aids for evaluating the severity of the disease. In recent years according to the results of treatment of encephalopathy with hypothermia, the incidence of the neurologic impairments in children seems to be significantly reduced. To better estimate the prognosis of HIE, it is necessary to be able to estimate the extent of injury during childbirth and even before, at the onset of labor.

Keywords: HIE, Newborn Encephalopathy

\section{-Monitoring of the Patient with Shock}

\section{Azita Behzad ${ }^{1, *}$, Seyedeh Masumeh Hashemi ${ }^{1}$, Seyedeh Narjes Ahmadizadeh ${ }^{1}$}

${ }^{1}$ Pediatric Intensive Care Department, Mofid Children Hospital, Shahid Beheshti University of Medical Sciences, Tehran, Iran

${ }^{*}$ Corresponding Author: Pediatric Intensive Care Department, Mofid Children Hospital, Shahid Beheshti University of Medical Sciences, Tehran, Iran. E-mail: azitab57@gmail.com

\section{Abstract}

Shock is a state of inadequate systemic perfusion and so severe tissue hypoxia. The goal of treatment is early reverse of deficit in perfusion and minimize complication such as multiorgan failure and death. In shock management, monitoring of response to resuscitation attempts are very important. For effective management, continuous hemodynamic status monitoring, frequent assessment of end organ perfusion and frequent adjustment of therapeutic interventions is vital. Improvement of clinical and physiologic signs of perfusion is monitored with invasive and noninvasive methods. Noninvasive assessment of systemic perfusion is done with interpretation of qualitative and quantitative information. For rapid qualitative information about the degree of systemic perfusion, Mental status and skin appearance are assessed. The quantitative assessment of perfusion includes noninvasive measures of the pulse rate, blood pressure, temperature, capillary refill time, urine output, Quality of central and peripheral pulses, auscultation of lung and heart sounds, pulse oximetry, End-tidal carbon dioxide (ETCO2) and Ultrasonic Cardiac Output Monitor (USCOM) in bedside. For invasive monitoring, Arterial pressure monitoring, Central venous pressure (CVP), Central venous oxygen saturation ( $\mathrm{ScvO2}$ ) and Lab tests (ex; lactate, anion gap) monitoring are done. We suggest that the optimal monitoring of the shocked child should include early 
and frequent measuring of all hemodynamic parameters above together to get therapeutic endpoints.

Keywords: Pediatrics, Shock Monitoring, Systemic Perfusion, Organ Perfusion

\section{- Problems and Solutions in the Field of Neonatal Research}

\author{
Hassan Boskabadi ${ }^{1}$ \\ ${ }^{1}$ Department of Pediatrics, Faculty of Medicine, Mashhad University of Medical Sci- \\ ences, Mashhad, Iran
}

\section{Abstract \\ The importance of research in increasing the level of neonatal health, which is the foundation of a healthy society in the com- ing decades, is not covered. The mere modeling of scientific re- sources, regardless of the real conditions of our country and our infants, has led to high costs for improving the health of infants and their low effectiveness. The main problems of neonatal re- search include: Research on current world issues, not our coun- try's problems, Research to produce an article, Lack of qualita- tive research on neonatal health, Lack of research in the field of quality of life. It is possible to correct the view of policy makers in the field of medical treatment and education, correct the view of research officials from producing articles to solving health problems, correct the view of key decision makers in the field of neonatology (board professors, neonatal association, organizers of important neonatal seminars, neonatal health department). Researching its direction, improving the infrastructure of re- search work, encouraging neonatal researchers to research the infrastructure and problems of our country, teaching how to re- search and empowering professors and students in directional and long-term research may lead to changes in neonatal health and decades Next, we will witness the flourishing and taste of re- search in increasing the health of our country's infants. \\ - Is Umbilical Cord Nucleated Red Blood Cell Count Related to a Premature Baby Prognosis?}

\author{
Hassan Boskabadi ${ }^{1}$, Forough Rakhshanizadeh ${ }^{2}$, \\ Maryam Zakerihamidi ${ }^{3}$
}

\footnotetext{
${ }^{1}$ Department of Pediatrics, Faculty of Medicine, Mashhad University o Medical Sciences, Mashhad, Iran. E-mail: boskabadih@mums.ac.ir

${ }^{2}$ Department of Pediatrics, Faculty of Medicine, Mashhad University of Medical Sciences, Mashhad, Iran. E-mail: RakhshanizafehF@mums.ac.ir

${ }^{3}$ Department of Midwifery, Faculty of Medical Sciences, Tonekabon Branch, Islamic Azad University, Tonekabon, Iran. E-mail: maryamzakerihamidi@yahoo.co.nz
}

\section{Abstract}

Background: Predictive value of the umbilical nucleated red blood cells (NRBCs) at birth for neonatal outcomes has been assessed. Hence, the present study was conducted to compare NRBC between discharged and dead neonates.

Methods: This cross-sectional study was performed on preterm newborns with a birth weight of $<1,500 \mathrm{~g}$ admitted to the Neonatal Intensive Care Unit of Ghaem Hospital, Mashhad, Iran, within 2012 - 2020. The subjects were divided into two groups of discharged and dead. Data collection tool was a researcher- made questionnaire containing three sections, namely maternal demographic information, neonatal data, and blood tests (measuring white blood cell [WBC], absolute NRBC, NRBC/100 WBC and blood gas). The collected data were analyzed in SPSS software (version 20), using t-test, Chi-square test, receiver operating characteristic curve, and regression models.

Results: A total of 205 neonates, including 136 discharged neonates (66.03\%) and 69 dead neonates (33.7\%), were examined in the present study. The results demonstrated a significant difference between the two groups in terms of the first minute Apgar score $(\mathrm{P}=0.023)$, fifth minute Apgar score $(\mathrm{P}=0.010)$, gestational age $(\mathrm{P}=0.000)$, birth weight $(\mathrm{P}=0.000)$, $\mathrm{WBC}(\mathrm{P}=0.020)$, absolute $\mathrm{NRBC}(\mathrm{P}=0.004)$, NRBC percentage $(\mathrm{P}=0.001)$, duration of mechanical ventilation $(P=0.029)$, duration of oxygen therapy $(\mathrm{P}=0.012)$.Moreover, mechanical ventilation $(\mathrm{P}=0.036)$, type of oxygen therapy $(\mathrm{P}=0.000)$, NRBC percentage $(\mathrm{P}=0.001)$, and absolute NRBC count $(P=0.001)$ showed a statistically significant relationship with neonatal survival rate.

Conclusions: As the findings indicated, mechanical ventilation, type of oxygen therapy, absolute NRBC count, and NRBC percentage can be used as markers for predicting neonatal mortality rate. Keywords: Neonates, Prediction, NRBC, Death, Discharged

\section{-New Biomarkers in the Diagnosis of Hypoxic Ischemic Encephalopathy}

\section{Hassan Boskabadi ${ }^{1}$, Maryam Zakerihamidi ${ }^{2}$}

${ }^{1}$ Department of Pediatrics, Faculty of Medicine, Mashhad University o Medical Sciences, Mashhad, Iran

${ }^{2}$ Department of Midwifery, Faculty of Medical Sciences, Islamic Azad University, Tonekabon Branch, Tonekabon, Iran

\section{Abstract}

Background: Precise and early diagnosis of asphyxia in neonates may improve the neonatal outcomes. Identifying diagnostic biomarkers of neonates at risk for brain damage, is the main goal of today's studies. Therefore, this study aimed to compare new biomarkers in the diagnosis of neonatal asphyxiation.

Methods: This prospective study was performed on 541 term infants including 266 (49.2\%) asphyxia (case group) and 275 (50.8\%) healthy subjects (control group) with accessible sampling. The data collection tool included a researcher-made questionnaire including maternal and neonatal characteristics, clinical and laboratory evaluation. Serum umbilical cord levels of biochemical markers including IL6, IL13, PAB, HSP 70 and NRBC levels in blood were determined. Data were analyzed by T-test, Chisquare, ROC and regression models.

Results: Variables interleukin-6 (IL6) $(\mathrm{P}<0.0001)$, IL1 $\beta$ ( $\mathrm{P}<$ $0.0001), \mathrm{PAB}$ (Proxidan antioxidant balance) $(\mathrm{P}<0.0001), \mathrm{Nu}-$ cleated red blood cell count (NRBC)/100WBC $(\mathrm{P}<0.0001)$, heat shock protein $(\mathrm{HSP} 70)(\mathrm{P}<0.0001), \mathrm{PH}$ the first hour of birth $(\mathrm{P}<$ $0.0001)$, BE the first hour of birth $(\mathrm{P}<0.0001), \mathrm{HCO} 3(\mathrm{P}<0.0001)$, first minute Apgar score ( $\mathrm{P}<0.0001)$, fifth minute Apgar score $(\mathrm{P}$ $<0.0001)$ in the two groups, the difference was statistically significant. In the diagnosis of asphyxia, the most sensitive marker (89\%) was IL1ß more than $2.39 \mathrm{pg} / \mathrm{mL}$ and HSP 70 upper thano.23 $\mathrm{ng} / \mathrm{mL}$ whileIL6 higher than $9 \mathrm{pg} / \mathrm{mL}$ determined as the most specific marker (85\%). For the diagnosis of asphyxia, combination of HSP + PAB and IL6 + lL1b + PAB + NRBC) $/ 100 W B C$ possesses the prediction power upper $93.2 \%$ and $87.3 \%$ respectively.

Conclusions: According to data analysis, the combination of new biochemical markers (NRBC count, IL6, IL1 $\beta$, PAB, HSP 70) could be a reliable marker for the diagnosis of infants with as- 
phyxia. The composition of the HSP + PAB indicators is more valid predictions $93.2 \%$ compared to the other combined indicators for the diagnosis of asphyxiated neonates. PAB values correlate with the severity of asphyxia.

Keywords: Asphyxia, Neonatal, Interleukin-6, Interleukin-IL1ß, Proxidan Antioxidant Balance, Nucleated Red Blood Cell Count, Heat Shock Protein

\section{nCovid-19 and Children with Renal Disease}

\section{Reza Dalirani ${ }^{1}$}

${ }^{1}$ Pediatric Nephrology Research Center, Mofid Children Hospital, Shahid Beheshti University of Medical Sciences, Tehran, Iran. E-mail: rdalirani@yahoo.com

\begin{abstract}
Covid-19 has a global spread. Children account for 1-5\% cases and have less severe disease compared to adults however preschool children and infants may have more severe disease. Are patients with kidney disease including children on dialysis, transplantation or immunosuppressive drugs at higher risk for Covid-19 infection? Comorbidity are common in patient with CKD and on dialysis or transplant recipients. Patients receiving therapy with drugs such as Corticosteroids, Calcineurin Inhibitors, Mycophenolate Mofetil, Azathioprine, Cyclophosphamide, Rituximab has been considered immunosuppressive. Decisions regarding change or reduced dose of immunosuppressive drugs should be based on disease severity, risk of disease flare and risk-benefit of therapy. Renal biopsy is performed for necessary condition such as allograft rejection, Rapidly Progressive Glomerulonephritis, Vasculitis or Interstitial Nephritis. Patients with Nephrotic syndrome should be treated with recommended dose of Prednisolone. Continue ACE Inhibitors or ARB in patient who are receiving them. In children with CKD Acetaminophen is safe, NSAID should be avoided, session of dialysis and CAPD should be continued. In patients with transplantation and mild Covid-19 infection immunosuppressive therapy continued, however in pneumonia due to Covid-19, Mycophenolate may be discontinued and reduction dose of Calcineurin Inhibitors have been considered. In conclusion Covid-19 is a mild disease in children with kidney disease and who are on dialysis or immunosuppressive therapy. However further study is required to confirmed these findings. Keywords: Children, COVID 19, Renal Disease
\end{abstract}

\section{-The Latest in Diagnosis of Chronic Asthma}

\section{Zahra Daneshmandi ${ }^{1}$}

${ }^{1}$ National Research Institute Tuberculosis and Lung Disease (NRITLD), Shahid Beheshti University of Medical, Tehran, Iran. E-mail: daneshmandiz@yahoo.com

\section{Abstract \\ Asthma is a global problem affecting millions of people all over the world. Monitoring of asthma both in children and in adult- hood is an indispensable tool for the optimal disease manage- ment and for the maintenance of clinical stability. The spirometry is the main test for detecting and measuring airway obstruction in children over 5 years old and adults and it has some precision for predicting future attacks. Spirometer parameters should}

be adjusted according to sex, age, and ethnicity. The diagnosis of asthma using spirometry involves interpreting the values of forced expiratory volume in the first second of expiration (FEV1), forced vital capacity (FVC), and the ratio of FEV to FVC obtained from the spirometer. Home monitoring of peak expiratory flow (PEF) may be use as an additional functional test in the monitoring of asthma. According with most of the guidelines, PEF measurement should not be routinely used to monitor asthma in children, unlike in adults where it is recommended for subjects with severe asthma or with poor perception of airflow limitation FeNO has a greatest use in bronchial asthma, particularly, it is considered a non-invasive biomarker to identify and to monitor airway inflammation but currently, there is not a consensus on the use of the FeNO in the management of asthma treatment. Fractional exhaled nitric oxide (FeNO) measurement correlates with eosinophilic airway inflammation and therefore with the most common asthma endotype, independently of gender, and age. Keywords: Asthma, Spirometry, FeNo, PEEF

\section{-Accuracy of Ultrasound in the Diagnosis of Skull Fractures in the Pediatric Emergency Department}

\author{
Hojjat Derakhshanfar ${ }^{1}$ \\ ${ }^{1}$ Research Institute of Children Health, Shahid Beheshti University of Medical Sci- \\ ences, Tehran, Iran
}

\section{Abstract}

Objective: The objective of this study was to identify the sensitivity, specificity, and predictive values of ultrasound for identifying skull fractures when compared to head CT scanning in pediatric patients with Head trauma.

Methods: The present study was a prospective cross-sectional observational study that was conducted over a six-month period. In this review, clinical ultrasound in the diagnosis of skull fracture, 320 children under 14 years with minor trauma the head, was used. A cranial CT scans was performed on the patients. The ultrasound results were compared to the results of head CT scans (which are considered the gold standard for diagnosing skull fractures) for sensitivity, specificity, positive predictive value, and negative predictive.

Results: The age range of the study population was from 24 days to 14 years with average of $4.73 \pm 3.29$ years. Cranial fractures were seen on the CT scan in 28 (8.75\%) of the 320 patients; 292 (91.25\%) CT scans were interpreted as normal. Emergency ultrasound performs with $85.71 \%$ sensitivity (95\% CI 75.3 - 99.5\%) and 100\% specificity (95\% CI 83.1-99.9\%) when compared to CT scan for the diagnosis of skull fractures. The positive and negative predictive values of emergency ultrasound for cranial fractures in our study group were 100\% (95\% CI 81.6 -100\%) and 98.64\% (95\% CI 82.1- 99.9\%), respectively. Conclusions: Ultrasound might be a reliable, fast, safe, and noninvasive initial diagnostic tool and can be an alternative primary technique in the diagnosis of skull fractures, especially in children. Keywords: CT Scan, Ultrasound, Head Trauma, Pediatric Patients

\section{-Comparing Emergency Residents with Pediatric Residents for Their Skills of ECG Interpretation}

\author{
Hojjat Derakhshanfar ${ }^{1}$
}


${ }^{1}$ Research Institute of Children Health, Shahid Beheshti University of Medical Sciences, Tehran, Iran

\section{Abstract}

Background: Electrocardiogram (ECG) is one of the most commonly performed investigations in cardiac diseases and ECG abnormalities can reveal the early manifestations of cardiac ischemia metabolic disorders, or life threatening dysrhythmias. ECG interpretation in pediatric medicine is used to diagnose many diseases. Pediatric Residents and EM Residents are usually the first to visit children and start treatments based on pediatrics ECG since due to differences in interpretation of Adult and pediatric ECG, we intended to evaluate the ability of EM Residents to interpret ate ECG and to compare it with that of pediatric residents, using various pediatrics ECG samples.

Methods: 80 participants including 39 pediatric residents and $41 \mathrm{EM}$ residents from two education hospitals of Shahid Beheshti university of medical sciences were enrolled in our study. A diagnosis test consisting of 10 ECG samples associated with a questionnaire containing questions about gender, academic year and passing of heart courses. It is study was conducted under the supervision of cardiologist and an emergency specialist. Who supervised the pediatric ECG selection, answers and scoring of each ECG. The score for each ECG was +1 which were given to a completely correct diagnosis and any point was given if the answer was wrong or any differential diagnosis was mentioned after the test, the answer sheets were collected and were analyzed with SPSS program two of study authors who were kept blind to the read identities of participants.

Results: After classification of groups, the overall average score for so residents were $(5 / 2 \pm 1 / 25)$. Significant correlation was found bet ween the diagnosis score. The average score of EM Residents was (6/1 $\pm 1 / 12)$ and for pediatric residents was ( $4 / 4 \pm 0 / 64)$, respectively $(\mathrm{P}<0 / 001)$ in addition, there was a significant correlation between academic year and ability for interpretation of ECG. In order word interpretation of pediatric ECG skill was increasing with level of academic year $(\mathrm{P}=0 / 008)$.

Conclusions: Our study revealed that the overall score of ECG interpretation are low and the ECG interpretation which may have an important role in treatment children. Therefore, there is a need for more and better pediatric ECG training programs especially in cardiac emergencies.

Keywords: ECG Interpretation, Emergency Residents, Pediatric Residents

\section{- Fluid Challenge in children patients with COVID-19 Disease}

\section{Nasrin Esfandiar ${ }^{1}$}

1 Pediatric Nephrology Research Center, Research Institute for Children's Health, Shahid Beheshti University of Medical Sciences, Tehran, Iran

\section{Abstract}

COVID-19 Disease is a global problem that starts with fever, anorexia, malaise and cough. In severe cases needs ICU admission. Fluid therapy in critically children with COVID-19 Disease is a concern. Conservative fluid administration with crystalloid solution is recommended. Bullous fluids are considered only if patient is fluid responsiveness based on clinical, paraclinical and laboratory parameters such as passive leg raising and stroke volume variations. The aim of this review is to study fluid therapy in critically ill pediatric patients with COVID-19 Disease admitted to ICU.

Keywords: COVID-19; SARs-CoV-2, Fluids, Child, Intensive Care

\section{-Adolescent's Spiritual Health in COVID-19 Pandemic: A Qualitative Study}

\section{Azam Shirinabadi Farahani ${ }^{1}$}

${ }^{1}$ Department of Pediatric Nursing, School of Nursing and Midwifery, Shahid Beheshti University of Medical Sciences, Tehran, Iran

\section{Abstract}

Background \& Objective: Health is a concept that has different dimensions. One of its dimensions is spiritual health, which is often overlooked, while it is always necessary, especially in crises. In this study, the concept of spiritual health in children in the COVID-19 pandemic has been investigated.

Methods: This qualitative study was conducted in Tehran using content analysis. Data were collected through face-to-face interviews with 12 adolescents who had recently been released from home quarantine on suspicion of coronavirus infection. Data were analyzed using qualitative content analysis.

Results: Data analysis on the concept of spiritual health led to emergence of three main categories of hope, faith and patience. Conclusions: Nurses, as care providers, play an important role in maintaining and promoting health in all their dimensions, and can also promote the spiritual health of adolescents as the future makers of the country by focusing on each of the extracted themes.

Keywords: Adolescent, Spiritual Health, Qualitative Study, COVID-19

\section{-Respiratory Distress in Neonates}

\section{Nazanin Farahbakhsh ${ }^{1}$}

${ }^{1}$ Department of Pediatric Pulmonology, Mofid Pediatrics Hospital, Shahid Beheshti University of Medical Sciences, Tehran, Iran. E-mail: nazanin26farahbakhsh@yahoo.com

\section{$\underline{\underline{A b s t r a c t}}$}

Respiratory distress (RD) in neonates may cause significant morbidities and mortalities. Many are preventable so early recognition, timely referral and appropriate treatment is essential. RD defines as presence of tachypnea, chest retraction, grunting, nasal flaring and/or cyanosis in a neonate. Respiratory distress syndrome, transient tachypnea of neonates, meconium aspiration syndrome and pneumonia are the most common pulmonary causes of RD in neonates. However, the underlying etiology of $\mathrm{RD}$ in a neonate does not always present in the lungs and other systemic medical and surgical diseases may cause RD in a newborn. A detailed History and physical examination, clinical course and radiographic patterns may guide to identify the non-pulmonary causes, such as cardiovascular, neuromuscular, chest wall disorders. Regardless of the etiology, general management including IV fluid, oxygen therapy, respiratory support and maintaining vital signs should initiate as soon as possible to prevent significant complications

Keywords: Respiratory Distress, Neonates, Tachypnea 


\section{-Drug-Induced Pulmonary Toxicity: Lots of Drugs and Chemical Agents Can Cause Acute and Chronic Lung Injury}

\author{
Fariba Faranghi ${ }^{1}$ \\ ${ }^{1}$ Shahid Beheshti University of Medical Sciences, Tehran, Iran
}

\begin{abstract}
This article discussed some common causes of acute pulmonary toxicity: 1- Hypoventilation/ Respiratory suppression: The most common cause of pulmonary manifestation in acute drug poisoning is Respiratory suppression by CNS depressants mainly opium presented as bradypnea /hypopnea /apnea and cyanosis. Opioids toxidromes including CNS suppression/Respiratory suppression /Miosis, vomiting/pruritus, ileus confirmed by dramatic response to Naloxone, support this common and serious poisoning. Weakness of chest wall or diaphragm muscles is another cause of hypoventilation such as Elapid snakes envenomation, nicotinic or cholinergic Toxidrome (like organic phosphorus pesticides); 2- Aspiration pneumonia in comatose patients from unprotected laryngeal reflexes, secondary to vomiting as a common adverse effect of activated charcoal; 3- Direct toxicity from Hydrocarbons aspiration. Even small amount (1mL) of aliphatic Hydrocarbons like gasoline, with spirit can cause significant pulmonary injury including; pneumitis, air leak syndromes, pleural effusion, bacterial pneumonia; 4- Acute lung Injury/ ARDS: Rare in children including poisoning with opioids, salicylates, phosphine (Aluminum Phosphide), CO poisoning; 5- Simple asphyxiant gases: (Methane, Propane); 6- Toxic gas: Carbon monoxide poisoning: Subtle, common and fatal; 7- Impaired Airway Patency: Aspiration of caustic agents manifested as stridor/hoarseness/respiratory distress/tachypnea are grave symptom in this poisoning. Also, Airway Patency may impaired by bronchospasm, irritant gases (Ammonia, chlorine, SO2), increased secretions, laryngospasm etc; 8- Mixed etiology: Like organic phosphorus pesticides (bronchorrhea, muscle weakness, pulmonary edema); 9- Secondary to shock, cardiogenic pulmonary edema, infection

Keywords: Poisoning, Pulmonary, Children
\end{abstract}

\section{- Management of Shock in Multisystem Inflammatory Syndrome Associated with COVID-19 in Children (MIS-C)}

\author{
Seyedeh Masumeh Hashemi ${ }^{1, *}$, Azita Behzad ${ }^{1}$, \\ Seyedeh Narjes Ahmadizadeh ${ }^{1}$ \\ ${ }^{1}$ Pediatric Intensive Care Department, Mofid Children Hospital, Shahid Beheshti Uni- \\ versity of Medical Sciences, Tehran, Iran \\ *Corresponding Author: Pediatric Intensive Care Department, Mofid Children Hospital, \\ Shahid Beheshti University of Medical Sciences, Tehran, Iran. E-mail: seyedeh.ma- \\ sumeh.hashemi@gmail.com
}

\section{Abstract}

Severe inflammatory state shock is commonly encountered in critically ill patients due to multisystem inflammatory syndrome associated with COVID-19 (MIS-C) and is accompanied with high mortality. All four types of shock (i.e., distributive, cardiogenic, obstructive, and hypovolemic) have been observed in patients with MIS-C. Hyper-inflammatory immune response with cytokine release syndrome leads to loss of vasomotor tone and higher rates of mortality. In vasodilatory shock, norepinephrine is considered as the preferred vasopressor agent and as the second line of treatment, vasopressin or epinephrine could be added. In acute cardiac injury followed by ischemia, myocarditis or cardiomyopathy, inotropic agents such as milrinone, dobutamine and epinephrine may be contemplated and in patients with hypotensive cardiogenic shock, norepinephrine is administered as the first-line vasopressor. Pneumothorax, pneumomediastinum and rarely, cardiac tamponade are etiologies of obstructive shock in COVID-19. Hypovolemia may be present due to poor oral intake, high-grade fever, diarrhea and bleeding induced by anticoagulant medications. In pediatric patients presenting with COVID-19 and shock, we suggest parenteral fluid therapy with crystalloid solutions, $10-20 \mathrm{~mL} / \mathrm{kg}$ up to $40-60 \mathrm{~mL} /$ $\mathrm{kg}$ bolus infusion in settings with pediatric intensive care unit (PICU) availability. However, in settings with inadequate pediatric critical care services, it is recommended to administer bolus fluids $(10-20 \mathrm{~mL} / \mathrm{kg}$ per bolus, up to $40 \mathrm{~mL} / \mathrm{kg}$ ) only in hypotensive patient. Evidences of improved outcomes with early administration of vasopressors are signified recently. In conclusion, shock associated with COVID-19 seems to have better outcomes in children compared with adults.

Keywords: MISC, Shock, COVID 19

\section{-The Effect of Education on First Year Residents' Knowledge Regarding Hemovigilance System}

\author{
Mozhgan Hashemieh ${ }^{1, *}$, Khezr Doosalivand ${ }^{2}$ \\ ${ }^{1}$ Pediatric Hematologist and Oncologist, Imam Hossein Medical Center, Shahid Be- \\ heshti University of Medical Sciences, Tehran, Iran \\ ${ }^{2}$ Pediatrition, Ilam, Iran \\ *Corresponding Author: Pediatric Hematologist and Oncologist, Imam Hossein Medical \\ Center, Shahid Beheshti University of Medical Sciences, Tehran, Iran. E-mail: mozh- \\ ganhashemieh@gmail.com
}

\section{Abstract}

Background: Regarding the possible transfusion reactions, the knowledge of medical staff, especially clinicians, about blood transfusion guidelines and also complications have significant importance. Hemovigilance is a system that monitors, records, and evaluates all steps of whole transfusion chain including collection, transfusion, and undesirable effects of blood products. Therefore, this study was conducted with the aim of the effect of education on residents' knowledge about hemovigilance system. Methods: This interventional study (pre \& post) was conducted on 80 medical assistants in 2018. Each participant received a questionnaire including 20 multiple choice questions for evaluating their knowledge about hemovigilance, then an educational intervention was performed by presenting an educational booklet to them. After two weeks, all 80 subjects were tested again in the two groups (same questions and in the same way as before) and the test results of the two groups were compared. The data were collected by a valid and reliable questionnaire. The data were analyzed by SPSS 21 software, paired t-test, and descriptive statistics.

Results: The mean age of participants was $31.4 \pm 3.6$, and the score of assistants' knowledge was 9.2 and after the intervention were 13.7. The results showed that the intervention had a significant effect on their knowledge $(\mathrm{P}=0.0001)$. In the group that did 
not receive training, no significant change was observed in the knowledge status of residents and the score increased from 9.27 to $10.8(\mathrm{P}=0.9)$.

Conclusions: Periodical educational programs and publication of pamphlets are highly recommended for the assistants involved in blood transfusion.

Keywords: Hemovigilance, Knowledge, Education, Blood products, Transfusions

\section{- Pediatric feeding disorder; diagnostic and therapeutic challenges}

\section{Amirhossein Hosseini ${ }^{1}$}

${ }^{1}$ Pediatric Gastroenterology, Hepatology and Nutrition Research Center, Research Institute for Children's Health, Shahid Beheshti University of Medical Sciences, Tehran, Iran. E-mail:Amir1981hosseini@gmail.com

\section{Abstract}

Pediatric feeding disorder (PFD) is a common clinical diagnosis and is essentially the inability to orally consume an age-appropriate diet. Impaired structure and/or function of the gastrointestinal, cardiorespiratory, and neurological systems including various neurobehavioral conditions are associated with PFD. PFD should only be diagnosed in the presence of dysfunction of the medical (such as aspiration), feeding skill (such as need for texture modification of foods), nutritional (eg, concomitant malnutrition), or psychosocial (such as disruptive behaviors associated with feeding) domains. Thus, the common picky-eating toddler may or may not have PFD depending on whether there is associated dysfunction from picky eating. PFD, however, in cludes almost all gastrostomy tube-fed children and children with oropharyngeal dysphagia as well as many children with autism. Studies have shown that children with medical complexity have a higher prevalence of PFD. PFD is defined as impaired oral intake that is not age appropriate, and is associated with medical, nutritional, feeding skill, and/or psychosocial dysfunction. The proposed diagnostic criteria. PFD can be classified into acute ( $<3$ months' duration) and chronic (3 months' duration). Four important domains underlie PFD: medical, nutritional, feeding skills, and psychosocial. Because of interplay between these domains, impairment in one can lead to dysfunction in any of the others.

Keywords: Pediatric, Feeding Disorder, Diagnosis

\section{-Gastro esophageal Reflux Disease; diagnostic and therapeutic challenges}

\section{Farid Imanzadeh ${ }^{1}$}

${ }^{1}$ Pediatric Gastroenterology, Hepatology and Nutrition Research Center, Research Institute for Children's Health, Shahid Beheshti University of Medical Sciences, Tehran, Iran. E-mail: drimanzadeh@yahoo.com

\section{Abstract}

The passage of gastric contents into the esophagus is a normal physiologic process that occurs in healthy infants, children. Most episodes are brief and do not cause symptoms, esophageal injury, or other complications. In contrast, gastroesophageal reflux disease (GERD) is present when the reflux episodes are associated with symptoms or complications. The diagnosis of GERD is based primarily on clinical suspicion, which can be strengthened by additional diagnostic investigations that are aimed to quantify and qualify GERD. Other diagnostic interventions may be utilized to rule out conditions other than GERD in the presence of specific diagnostic clues. In the absence of a single 'gold standard' investigation to diagnose GERD in infants or children, the diagnostic tests discussed in this section should be seen in this light. Treatment options include: Non-pharmacological treatments: Lifestyle changes, including avoidance of specific foods and positional changes confer modest improvement in some reflux-related symptoms. Pharmacological treatments: Acid-suppressing medications are reasonably safe and effective for the treatment of established acid-related GERD. Proton pump inhibitor (PPI), as first-line treatment, is safe and recommended. If PPIs are not available or contra-indicated, H2RAs can be used. It is not recommended to use H2RA or PPI for the treatment of regurgitation/ crying/distress in otherwise healthy infants. A 4 - 8 week course of H2RAs or PPIs for treatment of typical symptoms (i.e. heartburn, retrosternal or epigastric pain) in children with GERD can be diagnostic and therapeutic.

Keywords: Gastro Esophageal Reflux Disease, Children, Diagnosis

\section{-Early and Family Based Intervention Influences on Child Development}

\author{
Narjes Jafari1,*, Farin Soleimani ${ }^{2}$ \\ ${ }^{1}$ Pediatric Neurology Research Center, Research Institute for Children's Health, Shahid \\ Beheshti University of Medical Sciences, Tehran, Iran \\ ${ }^{2}$ Pediatric Neurorehabilitation Research Center, Speech \& Language Pathology Depart- \\ ment, University of Social Welfare and Rehabilitation Sciences, Tehran, Iran \\ ${ }^{*}$ Corresponding Author: Pediatric Neurology Research Center, Research Institute for \\ Children's Health, Shahid Beheshti University of Medical Sciences, Tehran, Iran
}

\section{Abstract}

The prevalence of behavioral or developmental disorders is $11 \%$ to $20 \%$, in American children, and estimated percent in our country is the same. We have a golden period that environmental stimulus has an important and constant affect on brain development. - Newborn brain is composed of 100 million neurons.

- These migrate, organize, connect, and specialize in response to neurotransmitter systems.

- Newborns have 50 trillion synapses connecting these neurons at birth, and an explosion in synaptogenesis leads them to develop 20 times that number by their first birthday.

But this overproduction is followed by a period of synaptic pruning, so synapses that are used become stronger, and those neglected are pruned away, and usually, we have only half the number of 1 age synapses. So, let's claim: Environmental stimulus in early child hood shapes brain architecture. Parenting is the first important environmental factor that influence in early brain development. Toxic stress in this golden time influence on child development, cognitive and behavioral status, also increase disability and social problems, and finally early death, and a stable, loving and affectionate relationship, with parents, is the most critical protective familial factor. So, Pediatricians and other pediatric health care professionals have a critically important role to play in optimizing environmental influences on child development and behave, with: Promoting positive parenting, Screening for developmental and behavioral problems, Monitoring families for signs of parental stress, Family training and counseling.

Keywords: Development, Child, Environmental Factors 


\section{ABSTRACTS}

\section{-The Main Determinants of Speech Disorder in Children}

\author{
Narjes Jafari ${ }^{1}$ \\ ${ }^{1}$ Pediatric Neurology Research Center, Research Institute for Children's Health, Shahid \\ Beheshti University of Medical Sciences, Tehran, Iran
}

\begin{abstract}
Background: Determining the prevalence of speech disorders in children, especially identifying the risk factors and predictors associated with these disorders, is essential for determining the most appropriate approach. It is clear that in addition to hereditary backgrounds the child's family and social interactions also play a major role in these disorders.

Objectives: In this study, we aimed to evaluate the clinical, familial, and social parameters associated with speech disorders in children. Methods: Patients included 70 children with speech disorders aged 6 months to 6 years who referred to Shahid Beheshti University Comprehensive Developmental Disorders Center. The control group included 70 children in the same age group but without any speech disorders. A questionnaire investigate demographic characteristics, family history, perinatal characteristics and complications during childbirth, parental socioeconomic characteristics, parental social and family interaction characteristics, and relationship between parents and the child Results: Among children with speech disorders, isolated recessive communication impairment was reported in $11.4 \%$, simultaneous recessive communication impairment in $16 \%$, both cognitive and recessive impairments in $21.4 \%$ and expressive communication disorder and recessive impairments at $24.3 \%$. Comparison between the two groups showed a significant difference in male gender, parental education level, positive family history, adverse child social interactions and perinatal comorbidities.

Conclusions: Male gender, family history of speech disorder, inappropriate social interaction, pregnancy comorbidities, inadequate parental education, as cognitive impairment are associated with an increased risk of speech disorder in children. Keywords: Development, Child, Speech Disorder
\end{abstract}

\section{- Post COVID-19 Systemic Juvenile Idiopathic Arthritis in Children}

\author{
Vadood Javadi Parvaneh ${ }^{1}$ \\ ${ }^{1}$ Mofid Children's Hospital, Shahid Beheshti University of Medical Sciences, Tehran, \\ Iran. E-mail: vadoodj@gmail.com \\ https://orcid.org/0000-0002-4159-2052
}

\begin{abstract}
Background: COVID-19 has been reported to cause a variety of signs and symptoms during its three known phases. The hyperinflammatory state in the third stage of the disease can induce multisystem inflammatory syndrome in children (MIS-C)

Case presentation: We report a 7 years-old boy and a 2.5 years-old girl with COVID-19, which presented the pictures of Kawasakilike syndrome classified as MIS-C and a final diagnosis of systemic juvenile idiopathic arthritis (systemic JIA). The children had prolonger high-spiky-fever and systemic clinical features including arthritis, salmon-pink patches, serositis, generalized lymphadenopathy. This disease is an auto-inflammatory phenotype
\end{abstract}

with unknown etiology. Some environmental factors including viral infections have been proposed to trigger the disease in genetically susceptible children. So far, systemic JIA following COVID-19 has not been reported in the literature.

Conclusions: COVID-19 may trigger systemic JIA in children. Keywords: Systemic Juvenile Idiopathic Arthritis, Coronavirus Disease 2019, Children

\section{- Management of Children with Rheumatic Diseases in COVID-19 Outbreak}

\author{
Khosro Rahmani ${ }^{1}$, Vadood Javadi Parvaneh",* \\ ${ }^{1}$ Pediatric Rheumatologist, Mofid Children's Hospital, Shahid Beheshti University of \\ Medical Sciences, Tehran, Iran \\ ${ }^{*}$ Corresponding Author: Pediatric Rheumatologist, Mofid Children's Hospital, Shahid \\ Beheshti University of Medical Sciences, Tehran, Iran. E-mail: vadoodj@gmail.com \\ https://orcid.org/0000-0002-4159-2052.
}

\section{Abstract}

Coronavirus disease 2019 (COVID-19) is a pandemic has affected various aspects of human life. Rheumatic diseases are rare in childhood and the prevalence of COVID-19 in these children is unknown. Some clinical and laboratory features of COVID-19 are held in common with rheumatic diseases. Furthermore, the management of medications in rheumatic diseases during epidemics, exposure, and COVID-19 is an important matter. COVID-19 has been presented with a variety of presentations. Clinical features and diagnoses which may mimic rheumatic diseases in COVID-19 includes macrophage activation syndrome, thromboembolism, arthritis, pleural/ pericardial effusion, autoimmune hepatitis, kawasaki like syndrome, kawasaki disease shock syndrome, DIC like syndrome, skin small vessel vasculitis, Reynaud's phenomenon, Stroke, and encephalopathy. Some laboratory features which may resemble rheumatic diseases includes leucopenia; lymphopenia; thrombocytopenia; increased ESR, CRP, and LDH; elevated AST and ALT; prolonged PT, PTT, and INR; increased ferritin, d-Dimer, and TG; positive ANA, Anti SSA, Anti SSB, anti Scl-70, and anti U1-RNP; decreased C3, C4; and high level of serum IL-6. In the ongoing COVID-19 outbreaks, children with rheumatic diseases are theoretically in the high-risk group. They should continue their routine medications and vaccinations if they have a stable condition without COVID-19 or exposure. In the case of exposure or infection, the parents would hold the rheumatic disease medications and consult with the child's pediatric rheumatologist for deciding in details and planning. Keywords: COVID-19, Children, Iran, Rheumatic Disease

\section{-The Impact of Social Media \& Video Games on Neurological Development of Children}

\section{Mohsen Javadzadeh ${ }^{1}$}

${ }^{1}$ Shahid Beheshti University of Medical Sciences, Pediatric Neurology Research Center Mofid Children Hospital, Shahid Beheshti University of Medical Sciences, Tehran, Iran

\section{Abstract}

Background: While social media has become a part of everyday 
life, scientists are just beginning to investigate how social media and other digital media may physically impact the developing brain. Most kids today are plugged into devices like TVs, tablets, and smartphones well before they can even ride a bike, and we witness many parents in our daily practice who boost about how good is their child at smartphones and tablets. Of course, technology can be part of a healthy childhood, as long as this privilege isn't abused; so why to worry? There are many concerns.

Methods: The present discussion will deal with 3 main fields of social media: TV, Video games, and Internet.

Results: TV: there are 2 main concerns: 1 - The content of shows such as violence, poor eating habits, bad language, sexual content, and so on. 2- The quantity of screen time which may harm the physical fitness, physical imagination, and social interaction skills. Video Games: Some video games may help the development of fine motor skills and strategic planning. But many of the concerns about the negative effects of television also apply to video games. Internet: Internet has a significant potential for providing children and youth with access to educational information, and can be compared with a huge home library. But in addition to the concerns about the TV and video games, some of the drawbacks are: Lack of an editorial standards and a form of addictive behavior. Finally, access to scholarly and research work in internet is just a click away and it may hamper one's creativity. Conclusions: The above issues will be discussed in more details throughout the webinar.

\section{-An Introduction to Hepatic Ischemic Encephalopathy}

\section{Mohammad Kazemian ${ }^{1}$}

1 Neonatal Health Research Center, Research Institute for Children's Health, Shahid Beheshti University of Medical Sciences, Tehran, Iran. E-mail: kazemianm@sbmu.ac.ir

\begin{abstract}
Hypoxic-ischemic encephalopathy (HIE) is one of the more challenging subjects in neonatal-perinatal medicine. Because different metabolic and genetic disorders may have a similar presentation and there is an incentive to complain to gynecologists and neonatologists. Recently we used the term HIE and insisted on birth-asphyxia. If there are clinical and par clinical findings in favor of asphyxia, we may consider it's a possibility. These criteria are: 1- umbilical cord blood pH less than 7.00 and base deficit $\geq 12 \mathrm{mmol} / \mathrm{L} ; 2$ - Multiorgan involvement (failure); 3- Apgar score of 34 W.G.A. These criteria and other similar guidelines as proposed by perinatal centers are not a gold standard and clinical evaluation and MRI guide us towards diagnosis. The following investigations are recommended: evaluation of placenta and umbilical cord pathologies, CBC-Diff, ABG, electrolytes, Blood Sugar, Calcium, Magnesium, liver enzymes, Cretinine, and brain sonography. Brain MRI is the most sensitive imaging tool for detecting cortical and white matter injury. Other problems like deep gray matter lesions, arterial infarction, hemorrhage, developmental brain malformations, may be declared as an underlying cause of neonatal encephalopathy. Recently the use of magnetic resonance spectroscopy (MRS) can provide useful information regarding the prognosis of neonatal encephalopathy. Cranial sonography and brain CT scan are not sensitive as MRI. Lumbar puncture is useful to rollout intracranial infection because meningitis can mimic the signs and symptoms of neonatal encephalopathy.

Keywords: Asphyxia, Encephalopathy, Hypoxia, Neonate
\end{abstract}

\section{-Early Detection of Signs of Autistic Spectrum Disorder (ASD)}

\author{
Mojgan Khademi ${ }^{1}$ \\ ${ }^{1}$ Shahid Beheshti University of medical sciences, Tehran, Iran
}

\section{Abstract}

Autistic spectrum disorder (ASD) manifestations can be detected from the early months of life, with about $50 \%$ showing symptoms in the first year: For example, parents report vision or hearing problems in the first year of life, also there is evidence of deficits in social and communication from 6 months of age, and repetitive behaviors and difficulties in imitation is reported in the second year. Early diagnosis and treatment can improve the prognosis, and also can reduce the burden on families and society. Some of the early red flags that professionals must be aware are (from $\mathrm{CDC}$ ): No babbling or pointing or other gestures by the age 12 months; No single words by 16 months; No two-word spontaneous phrases by 24 months; Loss of language or social skills at any age; Lack of joint attention. Sometimes there is a significant temporal distance between early symptom detections by parents and diagnosis by professionals and as a result, delay in treatment is seen. Thus, education of professionals to have more attention to these symptoms and raising the awareness of parents about them are important and these are crucial measures we discuss. Keywords: Children, Autism, Diagnosis

\section{nCow's Milk Protein Allergy Diagnostic and Therapeutic Challenges}

\section{Katayoun Khatami ${ }^{1}$}

${ }^{1}$ Pediatric Gastroenterology, Hepatology and Nutrition Research Center, Research Institute for Children's HealthShahid Beheshti University of Medical Sciences, Tehran, Iran. E-mail: K_khatami@sbmu.ac.ir

\section{Abstract}

The most common food allergen in infancy is cow's milk protein. It has different clinical presentations. Blood in stool is one of the most common symptoms in otherwise healthy baby who has cow's milk protein allergy (CMPA). Nausea, vomiting, gastroesophageal reflux, colic, diarrhea, constipation, transient enteropathy, protein-losing enteropathy, enterocolitis syndrome and colitis are other gastrointestinal presentations of CMPA but none of them are specific for diagnosis. It has also respiratory and cutaneous symptoms and signs which defers depending on reaction type (IgE or non- IgE mediated). Although doubleblind, placebo-controlled food challenge has been regarded as the gold standard for diagnosis of CMPA, in clinical practice it is not always applicable due to the risk of allergic reactions. Skin-prick testing and serum specific IgE levels may be useful for the diagnosis of IgE-mediated CMPA, and patch test has shown some promise in diagnosis of non- Ig E mediated allergy made on clinical basis. For patients with unexplained persistent gastrointestinal symptoms or iron deficiency anemia, upper and/or lower endoscopies with multiple biopsies should be considered. For management of CMPA, cow's milk should be omitted from diet of infants and breastfed mothers. In formula fed infants hypoallergenic formula should be prescribed. Gastrointestinal symptoms such as chronic diarrhea usually resolve in 2 to 
4 weeks and responses for early reactions such as vomiting and atopic eczema will be seen in $1-2$ weeks. If there is no improvement in clinical symptoms, after this period of elimination diet, then other diagnosis should be considered.

Keywords: Cow's Milk Allergy, Infant, Diagnosis, Treatment

\section{-Acute Kidney Injury in Pediatric Patients with COVID-19}

\author{
Masoumeh Mohkam ${ }^{1}$ \\ ${ }^{1}$ Pediatric Nephrology Research Center, Research Institute for Children's Health, Sha- \\ hid Beheshti University of Medical Sciences, Tehran, Iran
}

\section{Abstract}

In December 2019, outbreak of coronavirus 2019 (COVID-19) disease occurred in Wuhan and rapidly spread to other areas. Nephrologic problems among patients with COVID-19 can manifest as acute kidney injury (AKI), hematuria, or proteinuria and some kind of complications like hypertension. AKI is largely due to hemodynamic changes and cytokine release but somehow due to the direct cytotoxicity of virus or drug nephrotoxicity. Patients with suspected or confirmed COVID-19 may present with different stages of acute kidney injury as part of their overall illness. The incidence of AKI in patients with COVID-19 was about 17 percent (range 0.5 to 80 percent) and approximately 5 percent of patients require renal replacement therapy (RRT). The incidence seems to vary by geographic location and proportion of critically ill patients included in each study. AKI was noted on admission or within 24 hours of admission in one-third of the patients. AKI correlated with severity of illness. For prevention of AKI in hospitalized patients, an emphasis should be placed on optimization of volume status to exclude and treat prerenal azotemia while avoiding hypervolemia, which may worsen the patient's respiratory status. Differences in management of AKI among patients with COVID-19 may include limited use of intravenous solutions. Most patients with COVID-19 characterized by respiratory problems have variable degree of oxygen requirements and/or airway control. Fluid resuscitation are understandably conservative as per acute respiratory distress syndrome criteria. Thus, fluid resuscitation should be individualized and based on trackable objective measures. The indications for RRT for AKI remain the same indications regardless of the COVID-19 status. Alterations in RRT that might be undertaken during the COVID-19 outbreak include continuous renal replacement therapy (CRRT) which remains preferred among critically ill patients with AKI and hypotension.

Keywords: Acute Kidney Injury, Children, COVID-19

\section{- Congenital Hypothyroidism}

\author{
Asieh Mosallanejad ${ }^{1}$ \\ ${ }^{1}$ Shahid Beheshti University of Medical Science, Tehran, Iran. E-Mail: mosalladr@ \\ sbmu.ac.ir
}

\begin{abstract}
Background: Congenital Hypothyroidism has been reported with a prevalence of 1/2000 to 1/4000 children worldwide. However, this disorder is more common in Iran $(1 / 640) \mathrm{CH}$ is classified to primary and secondary forms; primary $\mathrm{CH}$ may be permanent or transient. Diagnosis with newborn screening and prompt treatment can prevent short and long term complications.
\end{abstract}

Methods: In Iran and many other countries Congenital hypothyroidism is screened with TSH test. Blood from a heel-prick is routinely collected on filter paper cards. Suspected hypothyroid specimens are confirmed by intravenous free T4 and TSH testing. Results: Serum TSH, total and free T4 reduce to near normal range by 2 to 4 weeks. An elevated serum TSH and a low free T4 or total T4 confirm the diagnosis of primary hypothyroidism.

Discussion: Congenital hypothyroidism is a cause of treatable mental disability. Diagnosis of $\mathrm{CH}$ is based on thyroid function test result. Primary $\mathrm{CH}$ is highly prevalent in Iran, likely due to: iodine deficiency, consanguineous marriage and use of iodinecontaining drugs and etc. Early treatment with levothyroxine improves the neurological prognosis of patients. The goal of therapy is to bring T4 and TSH to the target range in order to normal growth and development.

Conclusions: To prevent of mental disability and growth retardation, it is important to diagnose and treat congenital hypothyroidism as soon as possible.

Keywords: Screening, Hypothyroidism, Disability

\section{-Varicella Zoster Virus Immunoglobulin $\mathrm{G}$ in Immunosuppressed Children with Acute Lymphocytic Leukemia Under Chemotherapy}

\section{Shiva Nazari ${ }^{1}$}

${ }^{1}$ Pediatric Congenital Hematologic Disorders Research Center, Research Institute for Children's Health, Shahid Beheshti University of Medical Sciences, Tehran, Iran

\section{Abstract}

Background: Varicella (chickenpox) is an exanthematic children's diseases caused by the varicella-zoster virus (VZV). There is a limited data available about VZV seroprevalence among patients with acute lymphocytic leukemia in Iran.

Methods: Thirty patients with acute lymphocytic leukemia were included in the current study. At the first step, clinical data and demographic information of patients were collected, and then 5 milliliters of serum sample were gathered from each patient. At the next step, the VZV IgG antibody was detected with the VARICELLA-ZOSTER ELISA IgG/IgM kit.

Results: Overall, serum samples were collected from 30 patients, including 16 males and 14 females. The median age of patients was 5 years (range, 2 months to 11 years). The VZV IgG antibody was detected in $23.3 \%(n=7 / 30)$ of included patients. Of all the patients with positive VZV IgG antibody, 4 (57.1\%) and 3 (42.9\%) were males and females, respectively. The highest frequency of VZV IgG antibody was seen in 2 - 5 year old children.

Conclusions: In general, a high prevalence of VZV IgG antibody was observed among pediatric patients with acute lymphocytic leukemia. Results showed that the immunization of immunosuppressed children with acute lymphocytic leukemia with liveattenuated VZV vaccine is required.

Keywords: Varicella Zoster, IgG Antibody, Acute Lymphocytic Leukemia, ELISA, Iran

\section{-Multi Organ Dysfunction in Infants with Hypoxic-Ischemic Encephalopathy}

\author{
Shamsollah Noripour ${ }^{1}$
}


${ }^{1}$ Neonatal Health Research Center, Research Institute for Children's Health, Shahid Beheshti University of Medical Sciences, Tehran, Iran. E-mail:sh.noripour@yahoo.com

\section{Abstract}

Fetal and neonatal acute hypoxic insult, affects all the vital organs of body beside the brain and nervous system, fortunately the multi organ involvement of body rather than the brain, are transient with no permanent sequala's. Multi organ system involvement include acute bowel necrosis, renal failure, hepatic injury, cardiac damage, respiratory complications or hematologic involvement. Then the laboratory assessment in theses regards is required over the early neonatal period. In the view of multiorgan involvement on Neonatal Encephalopathy, several scoring systems and criteria are introduced to evaluate the organ involvement as follow:

Renal: Anuria or oliguria $<1 \mathrm{~mL} / \mathrm{kg} / \mathrm{h}$ for $\geq 24 \mathrm{hr}$, Creatinine $>1$ $\mathrm{mg} / \mathrm{dL}$, proteinuria $\geq 2+$, Azotaemia $=$ BUN $>40 \mathrm{mg} / \mathrm{dL}$, Significant hematuria ( $>10 \mathrm{RBCs} / \mathrm{hpf}$ in centrifuged sample).

Cardiovascular: Hypotension treated with an inotrope for $>$ $24 \mathrm{~h}$, Systolic and or diastolic BP $<5$ percentile for age and sex, ECG or ECHO Abnormalities, Troponin > 1 ug/L, Elevated CK-MB isoenzyme.

Hepatic: SGOT or SGPT > 100, Elevation of AST, ALT, LDH to 1.5 times of upper normal level, Prothrombin activity $<60 \%$.

Respiratory: Need for ventilatory support with $40 \%$ oxygen for at least the first $4 \mathrm{~h}$ after birth, Requirement for intubation and Mechanical Ventilation $>48 \mathrm{~h}$ post birth.

Gastrointestinal: Gastric residuals, vomiting, abdominal distension/tenderness, gastrointestinal bleeding, evidence of $\mathrm{Ne}$ crotizing Enterocolitis.

Hematologic: Blood leucocyte count $<4500$ or $>30000 / \mathrm{mm} 3$, Thrombocytopenia (Platelet count $<100 \times 109 / \mathrm{L}$, APTT $>45$ s, Increase in nucleated RBCs to $\geq 26$ per100 WBCs

Central nervous system: Clinical evidence of neonatal encephalopathy, Abnormal Neurological exam, EEG abnormalities, Neuroimaging abnormalities.

Keywords: Multi Organ Dysfunction, Infant, HIE

\section{- Children and Their Parents in the COVID-19 Pandemic}

\author{
Manijeh Nourian ${ }^{1}$ \\ ${ }^{1}$ Department of Pediatric Nursing, School of Nursing and Midwifery, Shahid Behesht
} University of Medical Sciences, Tehran, Iran

\begin{abstract}
A holistic approach of the nursing profession has enabled nurses to identify the comprehensive needs of the child and family and to provide developmental and family- centered care. Since the beginning of the COVID -19 pandemic around the world, children and their parents have been exposed to a great deal of stress and their daily lives have changed dramatically. These vast changes, along with feelings of hopelessness and insecurity, pose a potential risk factor for children's growth and development. Factors such as high parental stress and parental anxiety due to Concerns about their child being infected with COVID-19, insomnia, school closures, excessive use of virtual space, unhealthy nutrition, social restrictions, reduced levels of physical activity, reduced social activities especially in adolescents, family economic problems and its negative consequences in creating unbalanced conditions in the family, can endanger the growth and development of the
\end{abstract}

child and the health of the parents along with creating difficult childhood experiences (ACEs). In this review study, the effects and outcomes of COVID-19 on different physical, psychological and mental dimensions of children and their parents are presented based on the studies that have been done in this field so far. Recog nizing the negative factors and effects of COVID-19 on the growth and development of children and parents' health, can help nurses and health care providers for implementing developmental care in children and families.

Keywords: COVID -19, Growth and Development, Parents, Child

\section{-Online Education in Children During COVID-19 Pandemic}

\author{
Parastoo Oujian ${ }^{1}$
}

${ }^{1}$ Pediatric Nursing Department, School of Nursing and Midwifery, Shahid Beheshti University of Medical Sciences, Tehran, Iran

\begin{abstract}
The Corona pandemic led to the closure of schools and educational institutions and limited access to education for children. Therefore, the educational approach has changed to e-learning. E-learning has advantages and disadvantages, among which the nursing advocate is crystallized. Inclusive development of education is not possible in all places, and this has challenged educational justice. Another important issue is the challenge of children with special needs during a pandemic. These children are exposed to many problems with the closure of educational centers and less visits to health centers. These challenges require careful planning and special attention to this group of children to minimize the adverse consequences of the disease on children.

Keywords: Online Education, Children, COVID-19 Pandemic
\end{abstract}

\section{-Kidney involvement \& hepatitis virus}

\section{Zahra Pournasiri}

${ }^{1}$ Pediatric Nephrology Research Center, Research Institute for Children's Health, Shahid Beheshti University of Medical Sciences, Tehran, Iran

\section{Abstract}

Viral syndromes are often implicated as probable triggers for autoimmune diseases. Researchers in pediatric cohorts also support this association in acute glomerulopathies. Renal disease may occur in $3 \%-5 \%$ of patients with chronic HBV infection. The pathologic manifestations of HBV-associated renal disease can be classified as those that occur as a result of either (1) immune-complex GN such as: membranous, membranoproliferative GN and IgA nephropathy) or (2) immune complex-related vasculitis (polyarteritis nodosa [PAN]). Acute kidney injury (AKI), which was once considered a rare complication of hepatitis A, has also increased. The risk factors for AKI with acute hepatitis A: revealed that smoking, diabetics, higher WBC count, ALT, and total bilirubin, prolonged prothrombin time, and lower albumin levels were independent predictors of AKI, AKI responds to conservative treatment in most cases. Causes: Dehydration due to pre-renal factors, such as vomiting and diarrhoea, Direct Nephrotoxicity of direct hyper bilirubinuria and bile salts, resulting in tubular dysfunction or acute tubular necrosis. Acute interstitial nephritis. Immune complexmediated nephritis. Massive intravascular hemolysis precipitated 
by viral hepatitis. Renal disease associated with hepatitis $C$ virus infection: Renal failure, membranoproliferative glomerulonephritis (MPGN), membranous nephropathy, polyarteritis nodosa (PAN), mixed cryoglobulinemia, Crescentic glomerulonephritis may be superimposed on any of these glomerular lesions, Focal segmental glomerulosclerosis (FSGS), In some patients, glomerular disease may be clinically silent.

Keyword: Viral Hepatitis, Hepatitis A Virus, Hepatitis C Virus, Hepatitis A Virus, Kidney

\section{- Renal Injury Induced by Animal Toxins}

\section{Zahra Pournasiri $^{1}$ \\ ${ }^{1}$ Pediatric Nephrology Research Center, Research Institute for Children's Health, Sha- hid Beheshti University of Medical Sciences, Tehran, Iran}

\begin{abstract}
Animal toxins are well recognized for their hazards to man. Nephropathy arising from animal origin includes toxins of snakes, scorpions, hymenoptera, jelly fish, grass carp (raw bile), spiders, caterpillars. Scorpion sting nephropathy including: Acute renal failure is the commonest nephropathy, Subnephrotic proteinuria, Vasculitis, Hemolytic-uremic syndrome (HUS) Hemoglobinuria, Acute tubular necrosis, Interstitial nephritis. Snake envenomation is a common cause of AKI. Factors associated with AKI: bite to hospital time, Hypotension, Albuminuria, Prolonged bleeding time, prolonged prothrombin time, Low hemoglobin and a high total bilirubin. Bee bite: The most renal injury is ATN following intravascular hemolysis and rhabdomyolysis. Also, these allergens can mediate various types of renal injuries, such as immune complex-mediated glomerulonephritis, renal microangiitis, or vasculitis, Acute interstitial nephritis. Spider bite: Renal involvement in spider bite is not frequent and mainly corresponds to an acute renal failure and rarely nephrotic syndrome. Key words: Bite, Animal, Snake, Scorpion, Bee, Spider, Kidney, Renal, Envenomation, Child
\end{abstract}

\section{- Neonatal Resuscitation: Initial steps}

\section{Mitra Radfar ${ }^{1}$ \\ ${ }^{1}$ Imam Hossein Medical Center, Shahid Beheshti University of Medical Science, Tehran, Iran}

\section{Abstract}

The primary goal of neonatal resuscitation at birth is to facilitate transition from intra uterine to extra uterine life. Pre-resuscitation Team Briefing demonstrates perinatal risk factors, the team leader, and the person responsible for writing the events. Postresuscitation team discussion updates data and facilitates making ideas for further improvement. An organized equipment check list should be used before every birth. A team with full resuscitation skills should be immediately available for every resuscitation. After birth, every baby should be evaluated rapidly for estimation of gestational age (preterm or term), breathing and active crying, and good muscle tone. Initial steps include: 1-Warm the baby, 2-Check the head and neck position to keep the airway open, 3-If needed, remove secretions from the airway, but not routinely and 4 - If the baby does not have adequate respira- tion up to this step, gentle stimulation can improve respiration. Then the newborn response to initial steps should be assessed. If the neonate does not have adequate spontaneous respirations or a heart rate of $\geq 100 \mathrm{bpm}$ within 1 minute of birth, positive pressure ventilation should be started, and immediate additional help should be requested. Ventilation is the most important action, and needs effective management during neonatal resuscitation. If persistent cyanosis is suspected, start free-flow oxygen with the blender set 21 to $30 \%$ oxygen and increase it as needed, and pulse oximeter should be used. If the baby has weak breathing efforts or persistently low oxygen saturation, CPAP will be helpful. The presence of meconium-stained amniotic fluid may increases the risk of neonatal compromise that needs advanced resuscitation. Remember that routine intubation for tracheal suction is not indicated. For non-vigorous newborns delivered through MSAF who have evidence of airway obstruction during PPV, intubation and tracheal suction can be beneficial. Keywords: Neonatal Resuscitation, Ventilation

\section{-Anti-Microbial Effect of Nigella Sativa Seed Extract Against Staphylococcal Skin Infection}

\author{
Shiva Rafati', Mohammad Niakan², Mohsen \\ Naseri $^{3}$ \\ ${ }^{1}$ Department of Pediatrics, Faculty of Medicine, Shahed University, Tehran, Iran \\ ${ }^{2}$ Department of Microbiology, Faculty of Medicine, Shahed University, Tehran, Iran \\ ${ }^{3}$ Department of Pharmacology, Faculty of Medicine, Shahed University, Tehran, Iran
}

\section{Abstract}

Background: The development of microbial resistance to the antibiotic has become a serious problem and a real challenge facing patients suffering from infectious disease. Nigella Sativa seeds were used in traditional medicine for the treatment of a variety of infectious diseases.

Objective: In this study, the in vitro antibacterial effects of oily extract of Nigella Sativa seeds against staphylococcal skin infection in neonates have been investigated.

Methods: the staphylococcal microorganisms were collected from skin pustules of 20 neonates and used for standard bacterial dilution (0.5MF). For the assessment of the antimicrobial effects of Nigella Sativa oily extract with concentration of 33 percent prepared and for determining the antimicrobial effect of extract, a standard bacterial dilution 0.5 MF (McFarland) was prepared. The antimicrobial effect of Nigella Sativa seed oil was evaluated using dilutions of $0.5,0.25,0.125,0.625$ of the extract and the amount of MIC and MBC was determined.

Results: In the laboratory, MBC (minimum bactericidal concentration) and MIC (minimum inhibitory concentration) of Nigella extract for samples was 0.25 to $0.5 \mathrm{mg} / \mathrm{mL}$ and 0.6 to $21 \mathrm{mg} /$ $\mathrm{mL}$, respectively.

Conclusions: The black seed oil extract showed significant in vitro inhibitory effects on Staphylococcus bacteria. Keywords: Nigella Sativa, Staphylococcus, Neonate

\section{-Treatment of Perinatal Asphyxia in Term and Late Preterm Infants}

\section{Reza Saeidi ${ }^{1}$}


${ }^{1}$ Neonatal Health Research Center, Research Institute for Children's Health, Shahid Beheshti University of Medical Sciences, Tehran,Iran. E-mail: saeedir@mums.ac.ir

\section{Abstract}

The incidence of perinatal asphyxia is 2 per 1000 births in developed countries, but the rate is up to 10 times higher in developing countries. Of those infants affected, $15-20 \%$ die in the neonatal period, and up to $25 \%$ of survivors are left with permanent neurologic deficits. Treatment may also include the following: 1- Initial management: Cardiorespiratory stabilization and Maintenance of adequate ventilation (avoidance of hypoxemia or hyperoxia, hypercapnia, and hypocapnia). Ranges of pO2 are 60 to $80 \mathrm{mmHg}$ and for pCO2 35 to $45 \mathrm{mmHg}$; Avoidance of fluid overload especially in patients with SIADH or severe renal impairment; During the period of therapeutic hypothermia, enteral feedings are withheld (due to reduced intestinal function and metabolism) and TPN is used to provide adequate nutrition; Glucose infusion rates are adjusted to maintain glucose levels 72 to $145 \mathrm{mg} / \mathrm{dL}$; Empirical antibiotics are administered until culture results are known. 2- Anticonvulsant therapy: Phenobarbital is still the drug of first choice but some drugs, such as lidocaine as add-on therapy, are effective. In a study of term infants with asphyxia, high-dose phenobarbital $(40 \mathrm{mg} / \mathrm{kg}$ ) was associated with a significant reduction in severe neurodevelopmental disability. 3- Specific Neuroprotective Strategies: Therapeutic hypothermia within the first 6 hours after delivery is the standard treatment of HIE in term and late preterm infants. 4- Other Potential neuroprotective agents include: Calcium channel blocker (nicardipine), melatonin, erythropoietin (EPO), stem cell, N-acetylcysteine, allopurinol, selective NOS inhibitors, and noble gases, such as xenon and argon.

Keywords: Perinatal Asphyxia, Therapeutic Hypothermia, Hypoxic-Ischemic Encephalopathy (HIE)

\section{- Perianal Disease; Diagnostic and Therapeutic Challenges}

\author{
Aliakbar Sayyari ${ }^{1}$ \\ ${ }^{1}$ Pediatric Gastroenterology, Hepatology and Nutrition Research Center, Research In- \\ stitute for Children's Health, Shahid Beheshti University of Medical Sciences, Tehran, \\ Iran. E-mail: drsayyari@hotmail.com
}

\begin{abstract}
Children, just as adults, have a variety of common anorectal problems that can be quite bothersome. The presentation of these problems may be age-specific. Perianal disease in children is common and encompasses a broad spectrum of pathologic processes including fissures, fistulae, abscesses, hemorrhoids, rectal prolapse, pilonidal sinus, and pruritus ani. Occasionally, systemic illnesses such as inflammatory bowel disease may coexist. Abscesses, fistulas, and fissures appear more commonly in infants and young children, whereas hemorrhoids and pilonidal disease are more common in teens and young adults. Fissures often can be treated medically but may require surgical treatment with lateral internal sphincterotomy. Abscesses and fistulas are common in infant males, especially robust infants who are breastfed. They may resolve with medical therapy but anal fistulotomy is not infrequently required. Hemorrhoids are rare in young children but may be an issue for teenagers. Acute symptomatic lesions may require excision if local measures cannot control the symptoms. Finally, pilonidal disease is a difficult problem for the patient and the surgeon. Persistently symptomatic lesions demand some type
\end{abstract}

of surgical treatment but wound healing is poor in the intergluteal cleft region. More extensive procedures requiring the transfer of fasciocutaneous flaps may be necessary to provide definitive relief. Anorectal problems in infants and children are frequent and bothersome. Although most are not associated with tremendous morbidity, they can lead to much patient and parent anxiety as well as frequent medical consultation until the problem is successfully treated or resolves.

Keywords: Perianal Disease, Children, Infants, Treatment

\section{-Hyperthyroidism in Children and Adolescent}

\section{Marjan Shakiba ${ }^{1}$}

${ }^{1}$ Research Institute of Children Health, Shahid Beheshti University of medical science, Tehran, Iran

\section{Abstract}

Hyperthyroidism is not common in children. The disease causes many of the same symptoms that it does in adults but also has unique effect on growth and development in children. Graves disease is the cause of hyperthyroidism in $>90 \%$. Less common causes include autonomously functioning toxic nodules, transient hyperthyroidism during the early phase of Hashimoto thyroiditis followed by eventual hypothyroidism (hashitoxicosis), or adverse drug effects (eg, amiodarone-induced hyperthyroidism). In infants, symptoms and signs of hyperthyroidism include irritability, feeding problems, hypertension, tachycardia, exophthalmos, goiter, frontal bossing, and microcephaly. Other early findings are failure to thrive, vomiting, and diarrhea. In children and adolescents, symptoms of acquired Graves disease may include sleep difficulties, hyperactivity, emotional lability, marked decrease in concentration and school performance, heat intolerance, diaphoresis, fatigue, weight loss tremor, and palpitations. Signs include diffuse goiter, tachycardia, and hypertension. Graves ophthalmopathy occurs in up to one third of children. Although eye findings are less dramatic than in adults, children may have eyelid lag or red or prominent eyes, sometimes with proptosis (exophthalmos). Children and adolescents may present with alterations in growth, including growth acceleration and advanced bone age; however, puberty is often delayed rather than precocious. Acute thyroiditis manifests with sudden onset of symptoms of hyperthyroidism, tenderness over the thyroid gland, and fever. About $10 \%$ of patients with acute thyroiditis have hyperthyroidism. Many have leukocytosis with a left shift. In subacute thyroiditis these manifestations are present but less severe and may have been preceded by a viral illness.

Keywords: Hyperthyroidism, Graves's Disease, Subacute Thyroiditis, Acute Thyroiditis

\section{- Immune Causes of Thrombocytopenia in Children}

\section{Bibi Shahin Shamsian ${ }^{1}$}

${ }^{1}$ Pediatric Congenital Disorders Research Center, Shahid Beheshti University of Medical Sciences, Tehran, Iran. E-mail: shahinshamsian@gmail.com

\section{Abstract}

Thrombocytopenia is defined as a platelet count of $<150,000$ 
micro-L. Thrombocytopenia should be suspected in any child presenting with a history of easy bruising or bleeding or petechiae, but it also may present as an incidental finding in an asymptomatic individual. The Etiology of Thrombocytopenia in children may be: Productive, destructive, hereditary, acquired, immune, non-immune A thorough history, physical examination and use of laboratory testing can lead to the appropriate diagnosis in most patients who have thrombocytopenia. One of the most important reasons of thrombocytopenia in children are immune disorders. They include: primary immune deficiencies (common variable immune deficiency-vid, Wiskott Aldrich Syndrome-WAS), collagen vascular disorders (Systemic Lupus Erythematosus-SLE), autoimmune lymph proliferative disorder (auto immune lymphoproliferative syndrome-ALPS) immune thrombocytopenia in neonates (fetal \& neonatal Immune thrombocytopenia). Awareness of rare/ Less common disorders with thrombocytopenia is the main challenge for improving diagnosis, with an ongoing need for education of pediatricians and haematologists, to whom patients are most likely to first present.

Keywords: Thrombocytopenia, Immune, Neonate, Alloimmune

\section{- Renal Manifestations of Viral Infections}

\author{
Mostafa Sharifian ${ }^{1}$, Nasrin Esfandiar ${ }^{1}$ \\ ${ }^{1}$ Research Institute of Children Health, Shahid Beheshti University of Medical Scienc- \\ es, Tehran, Iran. E-mail: sharifian.dorche@gmail
}

\section{$\underline{\underline{\text { Abstract }}}$}

Infections may directly invade kidney or cause immune mediated injury, antigens of microorganisms' form circulating immune-complexes what is seen in post infectious glomerulonephritis. Renal injury may occur in multi-organ failure as is the case in systemic inflammatory response syndrome (SIRS) or altered cytokine expression, hemodynamic instabilities, hemolysis, rhabdomyolysis, and cardio or hepatorenal syndrome. Nearly all viral infections can induce kidney injury by various mechanisms including direct cytopathic effects to immune complex mediated GN and vasculitis as well. Hepatitis B, hepatitis $C$, human immunodeficiency virus, hantavirus and coronavirus disease 2019 (COVID-19) infections can induce glomerulopathy. Many of the viral infections such as parvovirus, Epstein-Barr virus and cytomegalovirus have been associated with very severe injury in the form of collapsing focal segmental glomerulosclerosis. Clinicopathological manifestations of viral infections include; Acute kidney injury (AKI) which is the most common presentation and can be de novo or acute on chronic kidney disease, acute and chronic glomerulonephritis syndrome, nephrotic syndrome, nephritic-nephrotic syndrome, acute or chronic tubulointerstitial nephritis, and rapidly progressive glomerulonephritis. Up to forty percent of patients who recover AKI may develop chronic kidney disease (CKD). Renal involvement is common in patients with COVID-19 infections; patients can present with proteinuria and hematuria even in moderately involved patients and at hospital admission, but AKI frequently develops in critically ill patients and is a marker of multiple organ dysfunction and ominous outcome. Fluid resuscitation at emergency department and lung-protective measures lowers the risk of AKI by obviating ventilator-induced hemodynamic disturbances and cytokine storm effects on the kidneys. Keywords: Viral Infection, Kidney Injury, Proteinuria, Hematuria, Children

\section{- Determination of Various Japanese Scores in Kawasaki Disease}

\author{
Reza Shiari ${ }^{1, *}$, Niloufar Shahaani ${ }^{1}$, Mehrnoush \\ Hassas Yeganeh ${ }^{1}$, Khosro Rahmani¹, Vadood \\ Javadi Parvaneh ${ }^{1}$, Leila Ghasemi ${ }^{1}$ \\ ${ }^{1}$ Research Center of Children Health, Shahid Beheshti University of Medical Sciences, \\ Tehran, Iran \\ ${ }^{*}$ Corresponding Author: Research Center of Children Health, Shahid Beheshti University \\ of Medical Sciences, Tehran, Iran. E-mail: shiareza@yahoo.com
}

\section{Abstract}

Background: Kawasaki disease (KD) is a systemic vasculitis that occurs mostly in young children. KD affects the middle-size arteries, especially the coronary arteries and without sufficient treatment, it may cause coronary artery aneurysm in $25 \%$ of patients. The purpose of this study was to investigate the relationship between three most practical Japanese score (Kobayashi, Sano, and Egami) with coronary artery aneurysm in KD patients during the last ten years and to identify risk factors of coronary artery aneurysms.

Methods: Medical records of 363 Kawasaki patients referred since April 2008 were reviewed. Patients' demographic data and Kobayashi, Sano, and Egami scores of each patient were calculated. Based on echocardiographic findings, cases of coronary artery aneurysm were determined. Sensitivity, specificity, positive and negative predictive value, and the accuracy of each criterion were determined to predicting IVIG resistance and detect coronary artery aneurysm.

Results: There was a slight relationship between IVIG-resistance in Kawasaki children and its prediction based on the Kobayashi risk score, but no relationship was found between the Egami and Sano criteria. Sixty-three patients (17.4\%) had coronary artery lesions on time of diagnosis. There were no statistically significant differences between gender and mean age of children with and without coronary aneurysm. Also, there was no significant relationship between coronary artery aneurysm in Kawasaki children and its prediction based on the above three risk factors.

Conclusions: Despite the low accuracy of the three above score to predictive of patients with IVIG resistance, it seems that the variables of age, duration of fever, and C-reactive protein (CRP) are more useful than other variables and may be utilized to evaluate patients by establishing a more appropriate cut-off point. Keywords: Kawasaki Disease, Japanese Scores, Children

\section{- Diagnostic Tests in Developmental Delays and Disorders in Children}

\section{Farin Soleimani ${ }^{1}$}

${ }^{1}$ Pediatric Neurorehabilitation Research Center, Speech \& Language Pathology Department, University of Social Welfare and Rehabilitation Sciences, Tehran, Iran. E-mail: Soleimani_farin@Yahoo.com

\section{Abstract}

The pregnancy problems and its continuation during the neonatal and infancy can impair brain development, attention, and early cognition. Developmental delay in the first years of life continues throughout life if there is no early diagnosis and intervention. The developmental potential of about 200 million children is not achieved by living in low-income and middle-income countries 
(LMICs) and has negative effects on academic achievement, adult earnings capacity, and parental ability for the next generation of children. The structure of child development is multifaceted and includes cognitive, motor, and social-emotional domains. Standard tools provide a way to measure the development of a child and compare it to a standard norm. In this context, the Bayley Scales of Infant and Toddler Development (Bayley III) is one of the diagnostic scales to measure developmental delay as a gold standard. The Bayley III is a test that measures development from neonatal period to 42 months. Its main goals are to identify children with developmental delays. Bayley III assesses the child's three developmental skills: cognitive, language, and motor. The cognitive scale evaluates information processing, play skills, counting and number skills. The language scale consists of receptive and expressive subtests that are used to assess communication skills. The motor scale consists of fine and gross motor subtests. Keywords: Bayley Scales of Infant and Toddler Development (Bayley III), Developmental Diagnostic Tests, Child Development, Infants, Toddlers, Measurement

\section{-Serum Electrolytes and Trace Elements Disorders in Viral Diseases (Covid-19)}

\author{
Hosseini Tabatabaei ${ }^{1}$ \\ ${ }^{1}$ Research Institute of Children Health, Shahid Beheshti University of Medical Scienc- \\ es, Tehran, Iran. E-mail: Mt.tabatabaei@sbmu.ac.ir
}

\begin{abstract}
The Coronavirus will induce a rapid change in nephrology. Nephrology will be stronger and better if we display the same solidarity we did while we fought the virus. Malnutrition and electrolyte imbalance can make dysfunction in the immune system and impairment of the immune system causes increasing the risk of infection. Dietary management is considered a strategy to minimize the potential risk of viral infections (COVID-19). Potassium: It has shown that COVID-19 patient's loss of potassium from urine so it causes hypokalemia in these patients. Sodium: The hyponatremia that mostly due to the SIADH, up to $12 \%$ of coronavirus patients have a low sodium level. Calcium: Coronavirus membrane fusion is associated with extracellular conditions. It has been observed that cellular ion conditions involve in the entrance of the virus. Magnesium: The role of magnesium in immune function is mediated by several mechanisms including immune cell adherence, antibody-dependent cytolysis, and IgM lymphocyte binding. Zinc: Zinc is an important trace element that has critical roles in the body, proliferation and function of NK cells, macrophages, neutrophils, T and B cells, and also the production of cytokines can be controlled by zinc. Selenium: In the structure of some enzymes and as well as vitamin E prohibits producing free radicals. Iron: Viral replication needs iron, a high level of iron may increase viral infections. Folic Acid: has an inhibitory function, it might be useful for the treatment of new coronavirus. Copper: Copper is an essential trace element in the body and the need for protecting DNA from oxidative stress. Keywords: Electrolytes, Trace Elements, COVID 19
\end{abstract}

\section{-A Brief Look at the Pediatric Integrative Medicine}

\author{
Saeed Sadr r, Hanieh Tahermohammadi ${ }^{2,}$, , Shahpar \\ Kaveh $^{3}$
}

${ }^{1}$ Department of Pediatrics Pulmonary Diseases, Mofid Children's Hospital, Shahid Beheshti University of Medical Sciences, Tehran, Iran. E-mail: ssadr54@yahoo.com ${ }^{2}$ Department of Traditional Medicine, School of Traditional Medicine, Shahid Beheshti University of Medical Sciences, Tehran, Iran.E-mail: dr.hmohammadi@sbmu.ac.ir

${ }^{3}$ Traditional Medicine and Materia Medical Research Center, Shahid Beheshti University of Medical Sciences, Tehran, Iran. E-mail: skaveh83@gmail.com

${ }^{*}$ Corresponding Author: Department of Traditional Medicine, School of Traditional Medicine, Shahid Beheshti University of Medical Sciences, Tehran, Iran. E-mail dr.hmohammadi@sbmu.ac.ir

\section{Abstract}

The National Center for Complementary and Integrative Health (NCCIH), as a subset of the National Institute of Health $(\mathrm{NIH})$, provides evidence-based complementary and alternative therapies that are outside of conventional medicine as an evidence-based healthcare approach. By 1998, more than $60 \%$ of American medical schools had included part of their curriculum in complementary and alternative medicine, and then the branch of integrative medicine was introduced to mean the use of complementary medicine alongside conventional medicine. In this field of medicine, the physician has a holistic approach to the patient and the discussion of treatment is not limited to the symptoms of the disease. From 2000 to 2002, the American Academy of Pediatrics (AAP), due to the increasing prevalence of chronic pediatric diseases, the great interest of families in complementary medicine treatments for their children, as well as the interest of physicians to receive training in this field, to address issues related to the use of complementary and alternative medicines in children and development of resources for training physicians, formed a special group of complementary and alternative medicine and in parallel, the pediatric integrative medicine program was introduced to promote evidence-based integrative medicine approaches in pediatric diseases. In 2012, a program called pediatric integrative medicine in residency (PIMR) entered in several universities, including Arizona, as part of the medical education curriculum, with the goals of prevention, context-centered, relationship-based, personalization of treatment, participation, ecologically sustainable, and evidenceinformed pays attention to children's health.

Keywords: Children, Pediatric, Integrative Medicine

\section{-Neonatal Mortality and Related Factors in Mahdiyeh Hospital through Seven Years}

Maryam Khoshnood Shariati', Naeeme Taslimi Taleghani ${ }^{1,2, *}$, Fares Najari ${ }^{3}$, Maryam Varzeshnejad $^{4}$

${ }^{1}$ Clinical Research Development Center, Mahdiyeh Educational Hospital, Shahid Beheshti University of Medical Sciences, Tehran, Iran

${ }^{2}$ Research Institute for Children's Health, Shahid Beheshti University of Medical Sciences, Tehran, Iran

${ }^{3}$ Department of Legal Medicine, Shahid Beheshti University of Medical Sciences, Tehran, Iran

${ }^{4}$ Department of Pediatrics, School of Nursing and Midwifery, Shahid Beheshti University of Medical Sciences

${ }^{*}$ Corresponding Author: Neonatal Health Research Center, Research Institute for Children's Health, Shahid Beheshti University of Medical Sciences, Tehran, Iran. E-mail: naeemetaslimi@sbmu.ac.ir

\section{Abstract}

Background: Neonatal mortality is about 45 percent of all child mortality under five years. NMR (Infant Mortality Rate) is one of 
the most important indicators of the evolution of countries in the world. In Iran, NMR decreased from 16 per 1000 to 9 per 1000 during the recent forty years.

Objectives: We performed this study to determine NMR in the NICU of Mahdiye hospital through 7 years.

Methods: This retrospective descriptive study done on newborns admitted in Mahdiyeh NICU from 21 March 2013-20 March 2020. All the neonates with gestational age more than 22 weeks included in this study and, stillbirths were excluded. We used SPSS 19 to analyze our data. Quantitative and qualitative variables exhibited as mean with standard deviation and frequency. Also, Fisher exact and Chi-square tests were used for categorical variables comparison.

Results: Out of 24049 newborns admitted in our NICU through 7 years, 755 infants (3.13\%) died. The most common causes of death respectively were Respiratory Distress Syndron (RDS) $20.1 \%$, prematurity with RDS $18.2 \%$, sepsis $13.5 \%$, asphyxia $7.1 \%$, congenital anomaly $6.3 \%$, alone prematurity $5.9 \%$, congenital heart disease (CHD) $4 \%$, Congenital Diaphragmatic Hernia (CDH) 2.8\%, necrotizing entro colitis (NEC) 2.1\% and metabolic disease $1.9 \%$. Conclusions: This survey shows that RDS with prematurity is the leading cause of death in our NICU that this finding is consistent with other studies. Therefore, it seems that prevention of prematurity and RDS still can reduce NMR. So, improving the perinatal care of mothers is ever important to decrease NMR. Keywords: Respiratory Distress Syndrome, Newborn, Mortality, premature

\section{-Chest Compression and Medications in Neonatal Resuscitation: 2020 American Heart Association Guidelines for Cardiopulmonary Resuscitation}

\author{
NaeemeTaslimi Taleghani ${ }^{1,2, *}$, Maryam Khoshnood \\ Shariati ${ }^{2}$, Minoo Fallahi ${ }^{1}$ \\ ${ }^{1}$ Research Institute for Children's Health, Shahid Beheshti University of Medical Sci- \\ ences, Tehran, Iran \\ ${ }^{2}$ Clinical Research Development Center, Mahdiyeh Educational Hospital, Shahid Be- \\ heshti University of Medical Sciences, Tehran, Iran \\ ${ }^{*}$ Corresponding Author: Research Institute for Children's Health, Shahid Beheshti Uni- \\ versity of Medical Sciences, Tehran, Iran
}

\section{Abstract}

Most neonates that have inefficient breath at birth will respond to the initial steps of resuscitation or effective positive pressure ventilation. Heart rate is best indicator of response to resuscitation. Pulse oximetry and Electrocardiogram (ECG) provide continuous heart rate evaluation in neonates requiring resuscitation. Expert opinion is that ECG should be applied when giving chest compressions for the rapid and reliable estimation of heart rate. If heart rate remains at fewer than $60 / \mathrm{min}$ despite sufficient ventilation for at least $30 \mathrm{sec}$, beginning chest compressions is advisable. The advantage of any concentration compared with $21 \%$ oxygen during chest compressions is uncertain but may be rational to use greater concentrations. It may be advisable to deliver 3: 1 compression after each ventilation while giving chest compressions. The two thumb encircling hands method for chest compression may have advantages because more enhance blood pressure and less provider fatigue. If the heart rate has not risen to more $60 / \mathrm{min}$ after effective ventilation and chest compressions, it may be reasonable to give epinephrine ( 0.01 to $0.03 \mathrm{mg} / \mathrm{kg}$ ) with every 3 to 5 minutes intervals. The most reliable medication delivery is umbilical venous catheter (Endotracheal route with dose of 0.05 to $0.1 \mathrm{mg} / \mathrm{kg}$ is an alternative way). A newborn with findings of blood loss that not response to epinephrine, may be response to volume expansion with normal saline or blood at 10 to $20 \mathrm{~mL} / \mathrm{kg}$. There is inadequate clinical evidence to determine what type of volume expander (crystalloid or blood) is more useful during neonatal resuscitation.

Keywords: Newborn, Cardiopulmonary Resuscitation, Chest Compression, Medications

\section{- Genetics of Kawasaki Disease in Iranian Children}

\author{
Mehrnoush Hassas Yeganeh, Khosro Rahmani, \\ Reza Shiari \\ ${ }^{*}$ Corresponding Editor: Mehrnoush Hassas Yeganeh
}

\section{Abstract}

Background: Kawasaki disease (KD)is the most common cause of acquired heart disease in infants and young children, may triggered by infectious agents, with the highest incidence in Asian countries. KD causes inflammation in the walls of medium-sized arteries and our major concern is coronary aneurysm. Kawasaki disease is also called mucocutaneous lymph node syndrome because it also affects lymph nodes, skin, and the mucous membranes. Several genotypes are identified as susceptibility genes for KD, none of them in Iranian population.

Objectives: Genetic determinants have been suggested to contribute to KD susceptibility. The risk of KD in siblings of affected children is ten times higher as compared with the general population and it is twice as high in patient born to parents with a history of KD compared with the normal population. Due to the racial differences in the frequency and significance of the identified genes, we aimed to investigate some possible genotypes in a sample of Iranian patients with the diagnosis of Kawasaki disease.

Methods: In this cross-sectional study, 100 children with KD who referred to Mofid Hospital from October 2015 to October 2016 were enrolled. 8p22-23-rs2254546 genotypes were analyzed using polymerase chain reaction (PCR) and direct sequencing techniques and the frequency of the three genotypes (GA, AA, and GG) were reported.

Results: Of 100 patients with a mean age of $1.9 \pm 1.7$ (0.1-10.2) years; $57 \%$ boys and $43 \%$ girls. $62 \%$ had GG, $30 \%$ GA, and $8 \%$ AA genotype. Conclusions:Themajorityof children withKDwereshowntohave this genetic susceptibility, which shows the importance of 8p2223-rs2254546 genotype in Iranian children. Future case-control studies can indicate the significance of these associations better. Keywords: Mucocutaneous Lymph Node Syndrome, Coronary Aneurysm, Genotype

\section{- Management of Spinal Muscular Atrophy New coordinated platform between patient and clinician in COVID-19 pandemic}

Marzieh Babaee ${ }^{1,2}$,S. Mansoor Rayegani', Parvaneh Karim Zadeh², S. Hasan Tonekaboni², Mohammad Mehdi Taghdiri², Mohamad Gofrani², Narjes Jafari², Mohsen Javad Zadeh² 
${ }^{1}$ Physical Medicine \& Rehabilitation Research Centre, Shahid Beheshti University of Medical Sciences, Tehran, Iran

${ }^{2}$ Pediatric Neurology Research Centre, Research institute for Children's Health. Shahid Beheshti University of Medical Sciences, Tehran, Iran

\section{Abstract}

Iran has a population of $\sim 83$ million people with marked cultural and economic differences. Due to culture and consanguineous marriage, inherited neuromuscular diseases (NMDs) are more common than some other countries. Iranian registry of SMA patients has been started with the onset of the outbreak of Covid-19. Due to the multi-organ involvement in COVID-19 infection and higher risks of mortality and morbidity for the neuromuscular disorders, offering rehabilitation and management of these patients especially SMA can be highly effective in the complications of the disease as well as the number of hospitalizations. With considering this situation, new platform for registry and management of SMA has been developed. In this platform, Tele rehabilitation as well as the creation of parent organizations, are contributing to the increased quality of the management of SMA patients. Over the past months, this care has provided new experiments. This article describes the outcome of this joint effort and shares the experience gained in this registry.

\section{- Provisional Tic Disorder}

\section{Ghazal Zahed ${ }^{1, *}$}

${ }^{1}$ Department of Child and Adolescent Psychiatry, Shahid Beheshti University of Medical Sciences, Tehran, Iran

${ }^{*}$ Corresponding Author: Department of Child and Adolescent Psychiatry, Shahid Beheshti University of Medical Sciences, Tehran, Iran. E-mail: ghazal.zahed@gmail.com

\begin{abstract}
A tic is when a part of the body moves rapidly, suddenly, repeatedly, involuntarily (motor tic) or sounds (such as barking, spitting) are produced involuntarily (vocal tic). Tics can be seen with any intensity. Most tics have a mild intensity, but they can be seen as severe and/or complex, which affect a person's function and life. Tics may seem like normal behaviors and are out of control, but one can stop them for a short time. Usually with age, the child notices their tics (many children over 10 years old) and the experience of relief immediately after, but overall, it is an uncomfortable experience for the child in the moment. The most common tic disorder is provisional tic disorder (transient tic disorder) that affects up to $24 \%$ of children. This group of tics are separate or multiple motor and/or vocal tics starting before the age of 18 , are temporary and disappear before one year, and do not occur due to the side effects of a substance or due to a physical disease. They also do not have diagnostic criteria for Tourette's disorder or persistent motor or vocal tic disorder. Common factors that exacerbate tics are fatigue, anxiety and excitement, watching TV or computer games, and taking certain medications. Treatment includes educating the child and parents about the nature of tics, educating the child to be more aware of the tics and substituting different behaviors, emotional support and the appropriate educational environment for them, and finally if necessary, prescription of medication.

Keywords: Provisional Tic Disorder, Child and Adolescent, Transient Tic, Treatment
\end{abstract}

\section{-Management of Pediatric Delirium}

\author{
Ghazal Zahed ${ }^{1, *}$ \\ ${ }^{1}$ Department of Child and Adolescent Psychiatry, Shahid Beheshti University of Medi- \\ cal Sciences, Tehran, Iran \\ *Corresponding Author: Department of Child and Adolescent Psychiatry, Shahid Be- \\ heshti University of Medical Sciences, Tehran, Iran. E-mail: ghazal.zahed@gmail.com
}

\section{Abstract}

Delirium is a neuro-cognitive disorder caused by a disturbance in the balance of neurotransmitters in the brain resulting in changes in the level and content of consciousness, disturbance of attention, awareness and cognition, and changes in behavior and emotion. Interventions of pediatric delirium include: Trying to minimize known risk factors; Encouraging parents to stay with their child and try to stimulate the five senses; Giving reassurance and improving orientation to time, place and person; Adjusting the light in the room so that it is known to the child when it is day and night time and preventing shadows from casting inside the room; Adjusting room temperature; Preparing favorite and familiar toys and belongings on the bedside; Providing eyeglasses and hearing aids if the patient has used it before; Encouraging the child to start moving; Adjusting drugs that make delirium worse and minimizing prescribed sedatives; Assessing the underlying physical causes that lead to this condition and treating underlying medical illnesses; Controlling of pain; Improving a patient's sleep at night and doing bedtime routines, and regulating sleepwake cycle. Despite protective measures, many times delirious children become severely agitated or anxious and show psychotic features (mostly hallucinations) and there is the danger of IV catheters or endotracheal tubes loss and harm to themselves so it is recommended to start medication to control these features. Typical antipsychotics, such as haloperidol and pimozide and atypical antipsychotics, such as risperidone are used in delirious children and adolescents. Sometimes it is necessary to correct sleep with medication.

Keywords: Delirium, Pediatric, Non-pharmacological Interventions, Pharmacological Treatment

\section{-Coping with the Child During COVID-19}

\section{Ghazal Zahed ${ }^{1, *}$}

${ }^{1}$ Department of Child and Adolescent Psychiatry, Shahid Beheshti University of Medical Sciences, Tehran, Iran

*Corresponding Author: Department of Child and Adolescent Psychiatry, Shahid Beheshti University of Medical Sciences, Tehran, Iran. Email: ghazal.zahed@gmail.com

\section{Abstract}

More than 10 months have passed since the COVID-19 pandemic in the world. Parents have complained about the behavioral and emotional changes in their children and feel it is difficult for them to manage these changes. Parents must consider these important tips: 1- Re-establishment of previous routines of life and letting children know that they are supposed to behave according to these routines (for example, waking up early and eating breakfast before the online classes start); 2 - Re-establishment of physical activity for their children. There may be restrictions on going to the gym and other public places for 
physical activity, but there is no justification for stopping these activities (conditions can be provided safely); 3- The child does not have the cognitive capacity to analyze some of these global issues, so parents should be careful not to allow the child to be exposed to all the catastrophic events of the environment in these critical conditions. These issues can be easily overlooked and can cause great harm to the child; 4- Considering the danger of Cyber addiction and Game addiction, during the COV-
ID-19 pandemic, children spend hours at the computer, and in addition to losing opportunities for socializing and physical activity, there is the risk of pathological dependence on cyber content and computer games, and encountering immoral content that is inappropriate for age, leading to changes in their child's mood and behavior and occurrence of psychiatric disorders.

Keywords: COVID-19, Coping, Child, Managing 


\section{INDEX}

$\rightarrow \mathrm{A}$

Ahmadizadeh Seyedeh Narjes

Alaei Fariba

$\mathbf{B}$

Babaee Marzieh

Bassir Mahta F.

Behzad Azita

Boskabadi Hassan

\section{D}

Dalirani Reza

Daneshmandi Zahra

Derakhshanfar Hojjat

Doosalivand Khezr

$>\mathbf{E}$

Esfandiar Nasrin

\section{$F$}

Fallahi Minoo

Farahbakhsh Nazanin

Faranghi Fariba

\section{G}

Ghasemi Leila

Gofrani Mohamad

\section{$\mathbf{H}$}

Hashemieh Mozhgan

Hashemi Seyedeh Masumeh

Hassas Yeganeh Mehrnoush

Hosseini Amirhossein

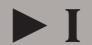

3

Imanzadeh Farid

$>$ J

17

Jafari Narjes

Javadi Parvaneh Vadood

$8,9,17$

9, 15

Javadzadeh Mohsen

9, 17

$\mathbf{K}$

Karim Zadeh Parvaneh

Kaveh Shahpar

Kazemian Mohammad

10

Khademi Mojgan

10

Khatami Katayoun

Khoshnood Shariati Maryam

16,17

6,15

17

6

7

15

17

7

3, 7

15,17

\section{M}

Mohkam Masoumeh

Mosallanejad Asieh

\section{$\mathbf{N}$}

Najari Fares

Naseri Mohsen

Nazari Shiva

Niakan Mohammad

Noripour Shamsollah

Nourian Manijeh

$>0$

Oujian Parastoo 


\section{INDEX}

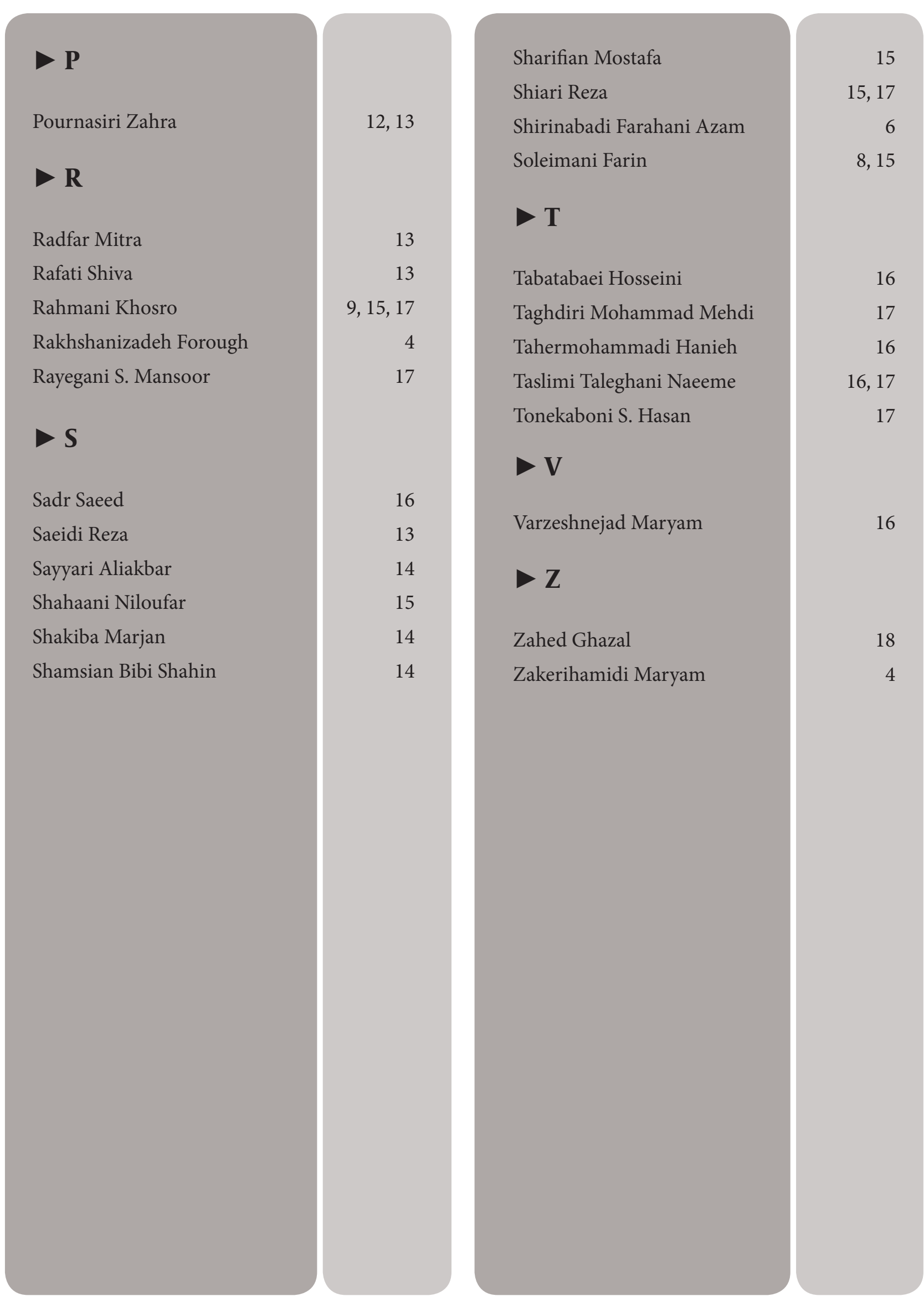




\section{LICENSE}

This is an open-access article distributed under the terms of the Creative Commons Attribution-NonCommercial 4.0 International License (http://creativecommons.org/licenses/by-nc/4.0/) which permits copy and redistribute the material just in noncommercial usages, provided the original work is properly cited. 
